\title{
In-cell structures of a conserved supramolecular array at the mitochondria-cytoskeleton interface in mammalian sperm
}

\author{
Miguel Ricardo Leung ${ }^{1,2}$, Riccardo Zenezini Chiozzi ${ }^{3,4}$, Marc C. Roelofs ${ }^{1}$, Johannes F. Hevler ${ }^{3,4}$, Ravi Teja Ravi ${ }^{1}$, Paula \\ Maitan $^{5,6}$, Min Zhang ${ }^{7}$, Heiko Henning ${ }^{5}$, Elizabeth G. Bromfield ${ }^{7,8}$, Stuart C. Howes ${ }^{1}$, Bart M. Gadella ${ }^{7}$, Albert J.R. Heck ${ }^{3,4}$, \\ and Tzviya Zeev-Ben-Mordehai ${ }^{1,2, *}$ \\ ${ }^{1}$ Cryo-Electron Microscopy, Bijvoet Centre for Biomolecular Research, Utrecht University, $3584 \mathrm{CH}$ Utrecht, The Netherlands \\ ${ }^{2}$ The Division of Structural Biology, Wellcome Centre for Human Genetics, The University of Oxford, Oxford OX3 7BN, United Kingdom \\ ${ }^{3}$ Biomolecular Mass Spectrometry \& Proteomics, Bijvoet Centre for Biomolecular Research and Utrecht Institute for Pharmaceutical Sciences, Utrecht University, 3584 CH \\ Utrecht, The Netherlands \\ ${ }^{4}$ Netherlands Proteomics Centre, $3584 \mathrm{CH}$ Utrecht, The Netherlands \\ ${ }^{5}$ Department of Clinical Sciences, Faculty of Veterinary Medicine, Utrecht University 3584 CM Utrecht, The Netherlands \\ ${ }^{6}$ Veterinary Department, Universidade Federal de Viçosa, 36570-900 Viçosa, Minas Gerais, Brazil \\ ${ }^{7}$ Department of Farm \& Animal Health and Biomolecular Health Sciences, Faculty of Veterinary Medicine, Utrecht University, 3584 CM Utrecht, The Netherlands \\ ${ }^{8}$ Priority Research Centre for Reproductive Science, Faculty of Science, The University of Newcastle, Callaghan, Australia, 2308 \\ correspondence to: z.zeev@uu.nl
}

\section{Summary}

Mitochondria-cytoskeleton interactions modulate cellular physiology by regulating mitochondrial transport, position- ${ }^{44}$ ing, and immobilization. However, there is very little struc- 45 tural information defining mitochondria-cytoskeleton inter- 46 faces in any cell type. Here, we use cryo-focused ion beam 47 milling-enabled cryo-electron tomography to image mammalian sperm, where mitochondria wrap around the ciliary cytoskeleton. We find that mitochondria are tethered to their neighbors through inter-mitochondrial linkers and are anchored to the cytoskeleton through ordered arrays on the outer mitochondrial membrane. We use subtomogram averaging to resolve in-cell structures of these arrays from three mammalian species, revealing they are conserved across 54 species despite variations in mitochondrial dimensions and 55 cristae organization. We find that the arrays consist of 56 boat-shaped particles anchored on a network of membrane pores whose arrangement and dimensions are consistent with voltage dependent anion channels. Proteomics and incell cross-linking mass spectrometry suggest that the conserved arrays are composed of glycerol kinase-like proteins. Ordered supramolecular assemblies may serve to stabilize similar contact sites in other cell types where mitochondria need to be immobilized in specific subcellular environments, such as in muscles and neurons.

sperm | mitochondria-cytoskeleton contact | cryo-electron tomography | cryo- ${ }^{65}$ FIB milling | cross-linking mass spectrometry | subtomogram averaging

42

actions with myofibrils and intermediate filaments (Milner et al., 2000; Stone et al., 2007). However, despite the prevalence of inter-mitochondria and mitochondria-cytoskeleton interactions and their integral roles in cellular function, there is very little information on the molecular architectures of these interaction sites in any cell type.

One of the most striking mitochondrial configurations occurs in amniote sperm, where mitochondria are arranged in a spiral around the axoneme, defining a region called the midpiece (Fawcett, 1970, 1975). Mitochondria are among the few organelles retained in sperm throughout their maturation, during which they otherwise lose most of their cytoplasm and organelles en route to becoming highly streamlined cells specialized for finding and fusing with the egg. The extensive mitochondrial sheath in amniote sperm may be an adaptation needed to power the large, long flagellum in these lineages. Variations in midpiece morphometry affect sperm motility and competitiveness (Firman and Simmons, 2010; Fisher et al., 2016), and different species rely on energy from mitochondrial respiration to different extents (Marin et al., 2003; Tourmente et al., 2015), warranting comparative studies of mitochondrial structure across species.

The core of the midpiece is the ciliary cytoskeleton, composed of the microtubule-based axoneme and accessory elements called outer dense fibers (ODFs). A poorlycharacterized network of cytoskeletal filaments called the submitochondrial reticulum lies between the ODFs and the mitochondria. The submitochondrial reticulum co-purifies with the outer mitochondrial membrane (OMM), suggesting that they are intimately associated (Olson and Winfrey, 1986, 1990). Mitochondria wrap around the cytoskeleton and are in turn ensheathed by the plasma membrane. As a consequence of this arrangement, each mitochondrion has three distinct surfaces (Olson and Winfrey, 1992) - one facing the axoneme, one facing the plasma membrane, and one facing neighboring mitochondria. Thin-section electron microscopy (EM) (Olson and Winfrey, 1992) and freeze-fracture EM (Friend and Heuser, 1981) suggest that each surface is characterized by a unique membrane protein profile. Notwithstanding the insight gained from these methods, such techniques require harsh sample preparation steps that can dis- 
tort fine cellular structure and limit achievable resolution (Al- ${ }_{139}$ Amoudi et al., 2004). As such, the molecular landscape of ${ }_{140}$ inter-mitochondrial and mitochondrial-cytoskeleton contacts 141 in the sperm midpiece remains largely unexplored.

Assembly of the mitochondrial sheath occurs late in 143 spermiogenesis and involves an intricately choreographed se- 144 ries of events (Ho and Wey, 2007; Otani et al., 1988). Ini- 145 tially, spherical mitochondria are broadly distributed in the ${ }_{146}$ cytoplasm. Mitochondria are then recruited to the flagellum, ${ }_{147}$ where they form ordered rows along the flagellar axis. Fi- ${ }_{148}$ nally, mitochondria elongate and twist around the axoneme. ${ }_{149}$ While our understanding of the molecular details of these ${ }_{150}$ processes is cursory at best, studies on gene-disrupted mice ${ }_{151}$ have implicated a number of proteins in mitochondrial sheath morphogenesis. For instance, mice expressing mutant forms ${ }_{153}$ of kinesin light chain 3 (KLC3) have malformed midpieces, ${ }_{154}$ hinting at a role for microtubule-based transport (Zhang et ${ }_{155}$ al., 2012). Another example are the voltage dependent anion ${ }_{15}$ channels (VDACs), which are highly abundant proteins that ${ }_{157}$ mediate transport of metabolites, ions, and nucleotides like ${ }_{158}$ ATP across the OMM (Colombini, 2012). Male mice lacking ${ }_{159}$ VDAC3 are infertile and their sperm cells have disorganized mitochondrial sheaths (Sampson et al., 2001), so VDACs ${ }_{161}$ may also have unappreciated roles in mitochondrial trafficking; indeed, KLC3 binds mitochondria through VDAC2 ${ }_{163}$ (Zhang et al., 2012). Similarly, disrupting sperm-specific isoforms of glycerol kinase leads to gaps in the mitochondrial ${ }_{165}$ sheath despite proper initial alignment of spherical mitochondria (Chen et al., 2017b; Shimada et al., 2019). Mice lacking ${ }_{167}$ spermatogenesis-associated protein 19 (SPATA19) (Mi et al., ${ }_{168}$ 2015) or glutathione peroxidase 4 (GPX4) (Imai et al., 2009; ${ }_{169}$ Schneider et al., 2009) also have structurally abnormal mitochondria.

Here, we use cryo-focused ion beam (cryo-FIB) millingenabled cryo-electron tomography (cryo-ET) to image the ${ }^{172}$ mitochondrial sheath in mature sperm from three mammalian ${ }^{173}$ species. We take advantage of the uniquely multi-scale ${ }^{174}$ capabilities of cryo-ET to unveil new aspects both of the ${ }^{175}$ overall organization of the mitochondrial sheath and of the ${ }^{17}$ molecular structures important for its assembly. We find ${ }^{177}$ that mitochondria are tethered to their neighbors through ${ }^{178}$ inter-mitochondrial linkers and to the underlying cytoskeleton through conserved protein arrays on the OMM. Subto- ${ }^{180}$ mogram averaging revealed that these arrays are anchored ${ }^{18}$ on a lattice of OMM pores whose arrangement and dimen- ${ }^{182}$ sions are consistent with VDACs. Proteomics and in-cell ${ }^{183}$ cross-linking mass spectrometry suggest that the arrays con- ${ }^{184}$ sist of glycerol kinase (GK)-like proteins. Our data thus show ${ }^{185}$ that although mitochondrial dimensions and cristae architec- 186 ture vary across species, the architecture of the mitochondria- ${ }^{187}$ cytoskeleton interface is conserved at the molecular level.

\section{Results}

Mitochondrial dimensions and cristae organization ${ }_{192}$ vary across species. We imaged the mitochondrial sheath 193 in mature sperm from three mammalian species, namely the 194 pig (Sus scrofa), the horse (Equus caballus), and the mouse 195
(Mus musculus) (Fig. 1). These species differ in terms of sperm size, motility patterns, and metabolism. To visualize the overall organization of the mitochondrial sheath, we imaged whole sperm with a Volta phase plate (VPP) (Danev et al., 2014; Fukuda et al., 2015). Neural-network based segmentation (Chen et al., 2017a) of the mitochondrial membrane allowed us to visualize mitochondrial organization in three dimensions (Fig. 1a-f).

To investigate variations in mitochondrial width along the midpiece, we first measured the width of each mitochondrion at multiple points along its length. We then divided mitochondria into groups based on their positions along the midpiece, as measured by their distance from the head (Fig. S1). The midpiece is $\sim 10 \mu \mathrm{m}$ long in both pig and horse sperm, but $\sim 20 \mu \mathrm{m}$ long in mouse sperm, so each group represents $\sim 2 \mu \mathrm{m}$ in the pig and the horse and $\sim 4 \mu \mathrm{m}$ in the mouse. We found that mouse sperm mitochondria are $\sim 1.5$ times wider than pig and horse sperm mitochondria overall (Fig. S1a). In all three species studied, most mitochondria in the middle $(\sim 60 \%)$ of the midpiece are crescent-shaped tubes (Fig. 1d-f) with consistent widths along their lengths (Fig. S1b). Mitochondria at the proximal end of the midpiece are larger than their more distal counterparts (Fig. 1a-c, S1a). Moreover, proximal mitochondria have more variable shapes, evidenced by greater variation in their widths at different point along their lengths (Fig. S1b). Because mitochondria wrap around the axoneme, variations in mitochondrial dimensions both across species and along the proximodistal axis of the flagellum affect the overall diameter and rigidity of the midpiece, likely fine-tuning the hydrodynamics of sperm motility.

To visualize the internal organization of sperm mitochondria in a near-native state, we imaged sperm thinned by cryoFIB milling (Fig. 1g-I). This revealed unexpected diversity in the internal ultrastructure of mitochondria across mammalian species, especially in terms of cristae morphology. Horse sperm mitochondria have an expanded intermembrane space and a condensed matrix (Fig. 1i-j). Mouse sperm mitochondria have an expanded matrix, with a narrow intermembrane space and thin cristae (Fig. 1k-l). Pig sperm mitochondrial morphology is intermediate (Fig. 1g-h), and although the mitochondrial matrix was dense, we could identify individual complexes that resembled ATP synthase on cristae of FIBmilled mitochondria (Fig. S2a-b), which was confirmed by subtomogram averaging (Fig. S2b').

Inter-species differences in cristae morphology correlate with measurements of matrix volume relative to mitochondrial volume (Fig. S2d). In this regard, horse sperm mitochondria resemble "condensed" mitochondria, which correlate with higher rates of oxidative activity in a number of different cell types, including developing germ cells, neurons, and liver (Hackenbrock, 1968; De Martino et al., 1979; Perkins and Ellisman, 2011). Indeed, horse sperm are dependent on oxidative phosphorylation (Davila et al., 2016), whereas pig (Marin et al., 2003) and mouse sperm (Mukai and Okuno, 2004; Odet et al., 2013) are thought to rely largely on a glycolytic mechanisms. 


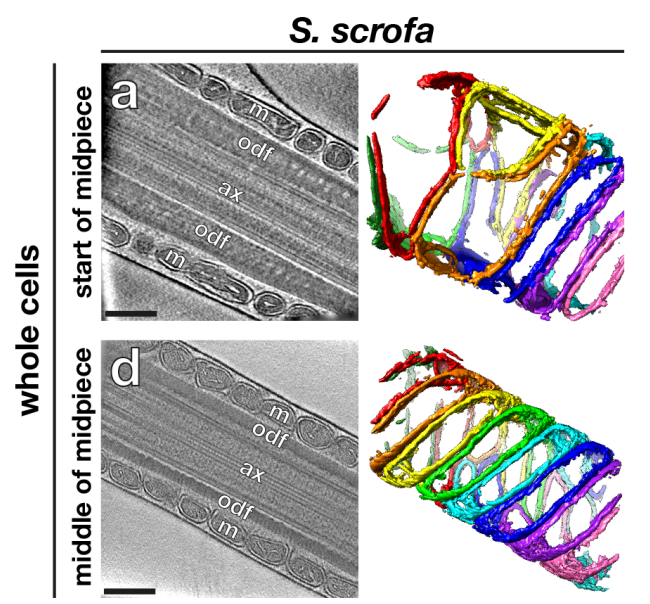

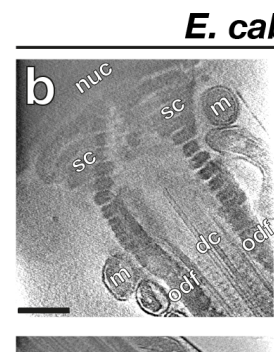

E. caballus
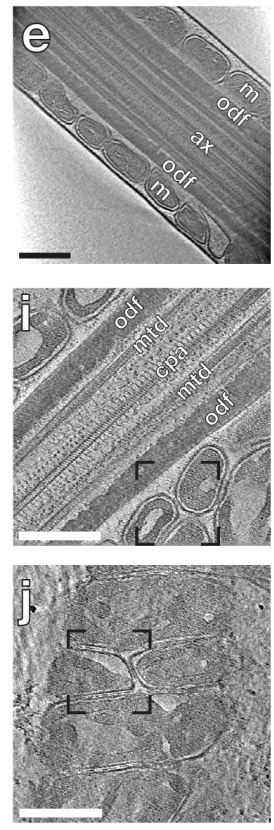
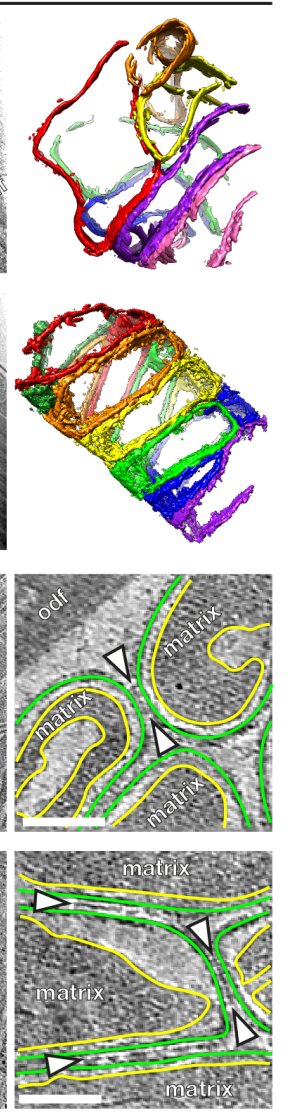

M. musculus
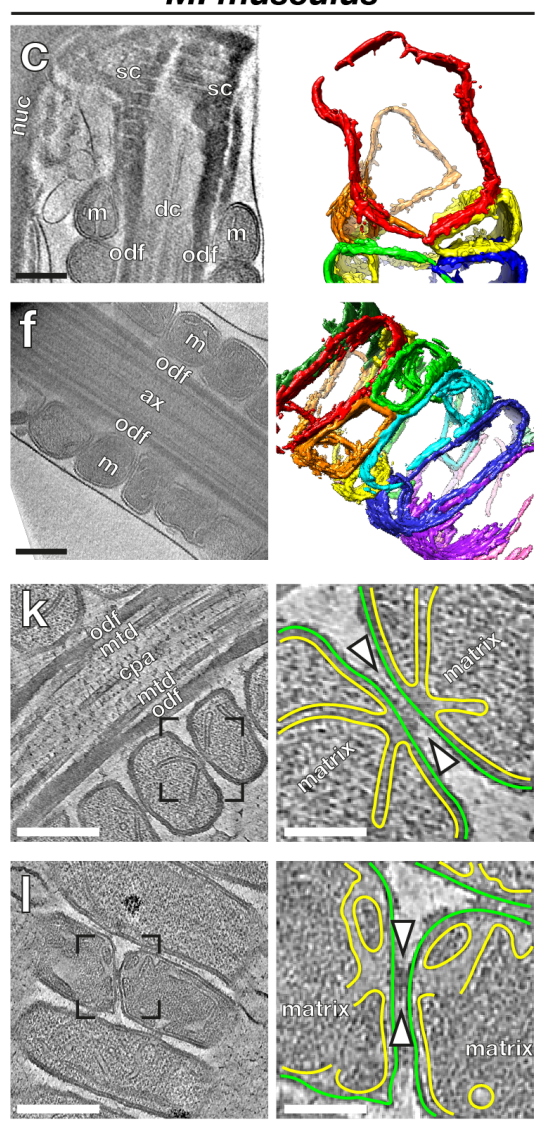

Fig. 1. Mitochondrial dimensions and cristae organization vary across species. (a-f) Slices through Volta phase plate cryotomograms (left) and corresponding three-dimensional segmentations (right) of mitochondria from the start (a-c) or middle (d-f) of the midpiece from pig $(a, d)$, horse $(b, e)$, and mouse (c,f) sperm. (g-l) Slices through cryo-tomograms of FIB-milled pig (g,h), horse (i,j), and mouse (k,l) sperm midpieces. Right panels show digital zooms of the regions boxed out in the left panels. The outer mitochondrial membrane is traced in green, the inner mitochondrial membrane in yellow, and the plasma membrane in blue. Arrowheads indicate inter-mitochondrial linker complexes. Labels: nuc - nucleus, sc - segmented columns, m - mitochondria, odf - outer dense fibers, dc - distal centriole, ax - axoneme, mtd - microtubule doublets, cpa - central pair apparatus, pm - plasma membrane. Scale bars: (a-I) left panels $-250 \mathrm{~nm},(\mathrm{~g}-\mathrm{l})$ right panels $-100 \mathrm{~nm}$.

Inter-mitochondrial junctions are associated with ${ }_{216}$ linker complexes. Mitochondria are closely packed within 217 the mitochondrial sheath, but it is unclear whether or how 218 individual organelles communicate with their neighbors. To 219 address this, we imaged inter-mitochondrial junctions cap- 220 tured in FIB-milled sperm lamellae. We observed trans- 221 mitochondrial cristae alignment in mouse sperm (Fig. 1k- 222 I), but not in pig or in horse sperm (Fig. 1g-j). Trans- 223 mitochondrial cristae alignment has also been observed in 224 muscle tissue of various organisms, and is proposed to medi- 225 ate electrochemical coupling between adjacent mitochondria 226 (Picard et al., 2015). To our knowledge, this is the first time this phenomenon has been observed in mature sperm from any lineage. It is particularly curious, however, that trans- ${ }^{222}$ mitochondrial cristae alignment in sperm is species-specific. ${ }_{229}$

We found that inter-mitochondrial junctions are charac- 230 terized by novel inter-mitochondrial linker complexes in all ${ }_{231}$ three species (arrowheads in Fig. 1g-l, Fig. S2c). These ${ }_{232}$ inter-mitochondrial linkers span the 8-nm distance between ${ }_{233}$ the outer membranes of neighboring mitochondria. In mouse ${ }_{234}$ sperm, these linkers are specifically associated with sites of trans-mitochondrial cristae alignment (Fig. 1k-l); in the pig and in the horse, they are positioned at regularly spaced intervals along inter-mitochondrial junctions (Fig. 1h-j). Electron-dense inter-mitochondrial junctions were also seen in cardiomyocytes by classical EM (Duvert et al., 1985; Huang et al., 2013; Picard et al., 2015). Thus, it is plausible that the as-yet-unidentified linker complexes we visualize here represent a more general structural mechanism for orchestrating inter-mitochondrial communication in various cell types.

\section{Ordered protein arrays at the mitochondria-cytoskele-} ton interface are conserved across species. To determine how mitochondria interact with the flagellar cytoskeleton, we imaged the mitochondria-cytoskeleton interface in cryo-FIB milled lamellae (Fig. 2). We found that the axoneme-facing surface of the OMM is characterized by an ordered protein array that is absent from the plasma membrane-facing surface (Fig. 2a). These arrays are present 


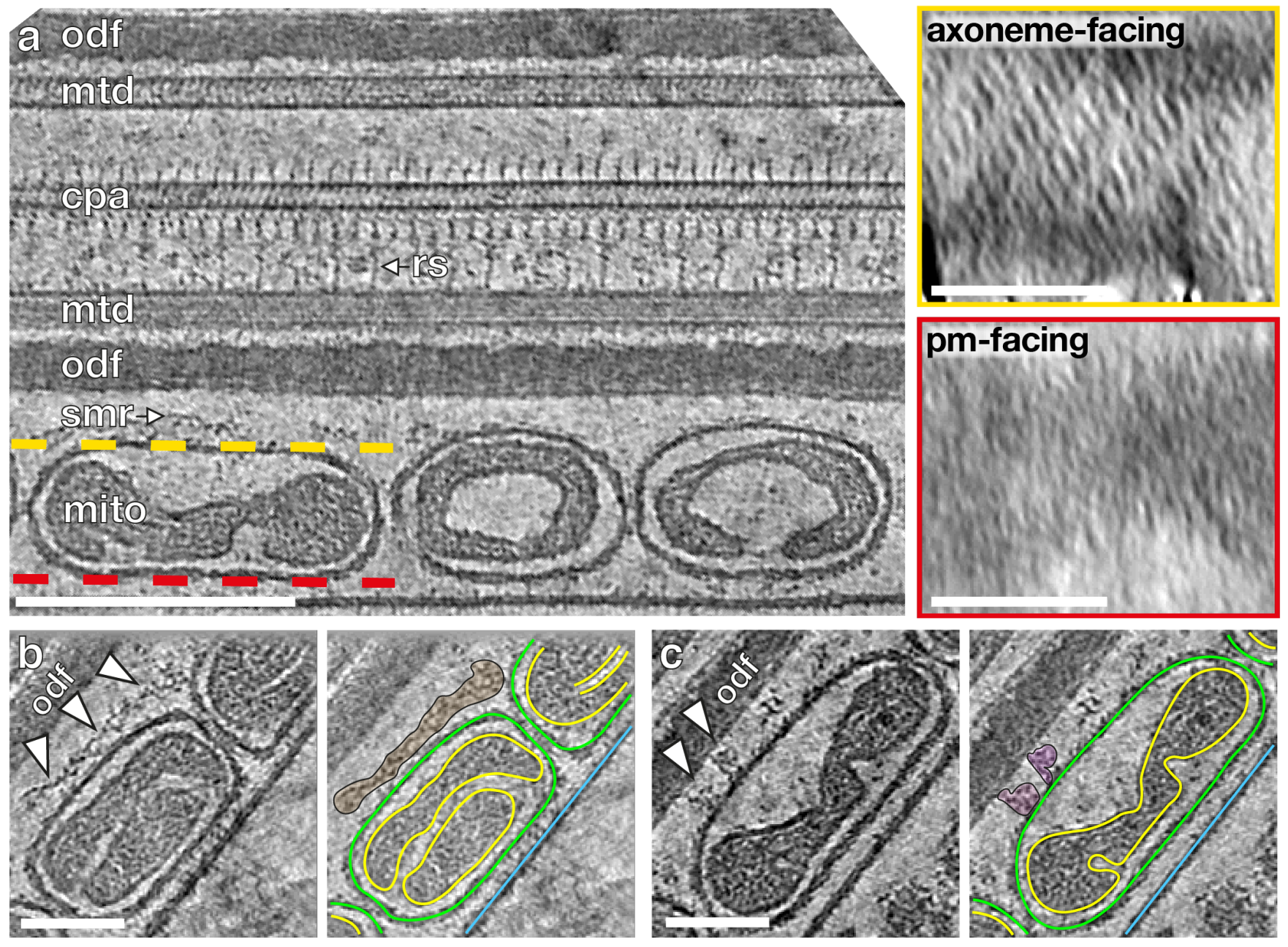

Fig. 2. Ordered protein arrays on the outer mitochondrial membrane directly interact with the submitochondrial reticulum. (a) Slice through a cryo-tomogram of a FIB-milled horse sperm midpiece, showing mitochondria (mito), the submitochondrial reticulum (smr) outer dense fibers (odf), microtubule doublets (mtd), and the central pair apparatus (cpa). Note how individual complexes (like the radial spoke, rs) are visible in the raw tomogram. The ordered protein array is only found on the axoneme-facing surface (yellow) of midpiece mitochondria, and not on the plasma membrane-facing surface (red). (b,c) Slices through a cryo-tomogram of a FIB-milled horse sperm midpiece showing how the array directly interacts with the submitochondrial reticulum to anchor mitochondria to the ciliary cytoskeleton (arrowheads). In right panels, the outer mitochondrial membrane is traced in green, the inner mitochondrial membrane in yellow, and the plasma membrane in blue. Scale bars: (a) left $-250 \mathrm{~nm}$, insets - $100 \mathrm{~nm}$; (b,c) $100 \mathrm{~nm}$.

in all three species and along the entire midpiece (Fig. S3a-f) 252 and resemble the particle rows seen on the axoneme-facing ${ }_{253}$ surface of the OMM in guinea pig sperm (Friend and Heuser, 254 1981) and in mouse sperm (Woolley et al., 2005) by freeze- 255 fracture EM. We observed direct interactions between the ar- 256 rays and cytoskeletal filaments surrounding the ODFs (Fig. 257 2b-c), indicating that these arrays tether mitochondria to the 258 midpiece cytoskeleton.

We then aligned and averaged sub-volumes containing 260 the protein arrays and the underlying OMM (Fig. 3, Ta- 261 ble S1). Our averages revealed 22-nm-long two-fold- 262 symmetric boat-shaped structures connected via four densi- 263 ties to a porous membrane (Fig. 3, Fig. S3g-i). Each boat- 264 shaped particle rises $\sim 5 \mathrm{~nm}$ above the membrane and consists 265 of two tilde-shaped densities arranged end-to-end. The boat- 266 shaped structures form rows in which each particle is related ${ }_{267}$ to its closest neighbors by a $\sim 10 \mathrm{~nm}$ translation perpendicu- 268 lar to the particle long axis and a $\sim 6 \mathrm{~nm}$ shift along this axis, yielding a center-to-center spacing of $\sim 12 \mathrm{~nm}$ (Fig. 3d-f). Each row is oriented $\sim 120^{\circ}$ to the long axis of the flagellum and adjacent rows are spaced $\sim 12 \mathrm{~nm}$ apart, forming extensive arrays on the axoneme-facing surface of the OMM (Fig. 3g). Remarkably, the averages we obtained from the three species were highly similar, both in terms of individual particle dimensions and in terms of their supramolecular arrangement (Fig. 3, Fig. S3). This conservation suggests that these arrays are a crucial structural element of the mitochondrial sheath.

Our averages revealed that the OMM underlying the protein arrays is studded with $\sim 3-4 \mathrm{~nm}$ pores arranged in a pseudo-lattice with a center-to-center spacing of $\sim 5 \mathrm{~nm}$. (Fig. 3a-c, Fig. S3g-i). These pore sizes are consistent with the diameters of the voltage dependent anion channels (VDACs), which are known to form ordered arrays in the 


\section{S. scrofa}
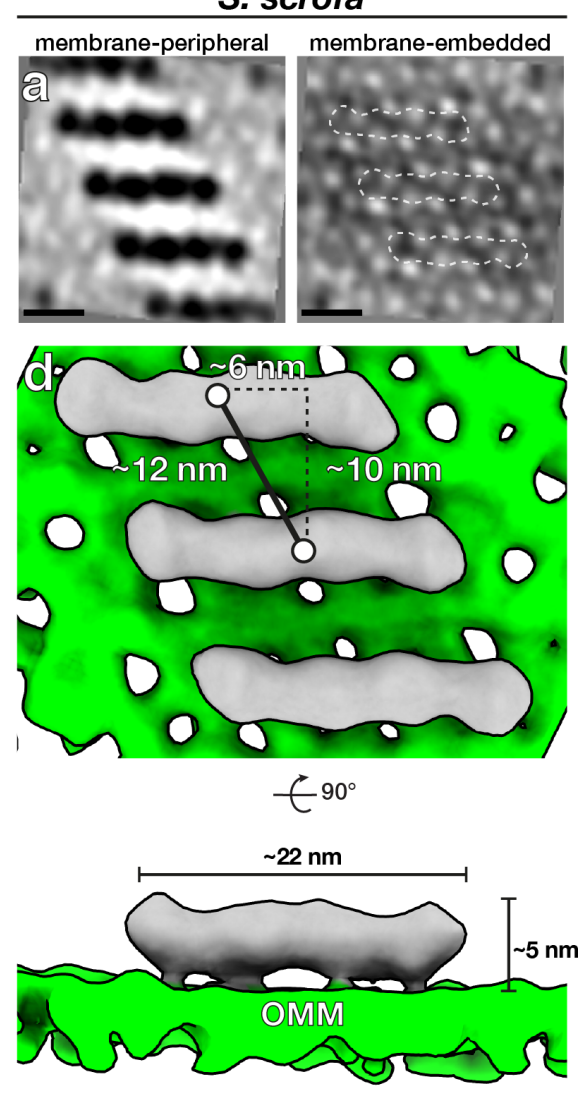

E. caballus
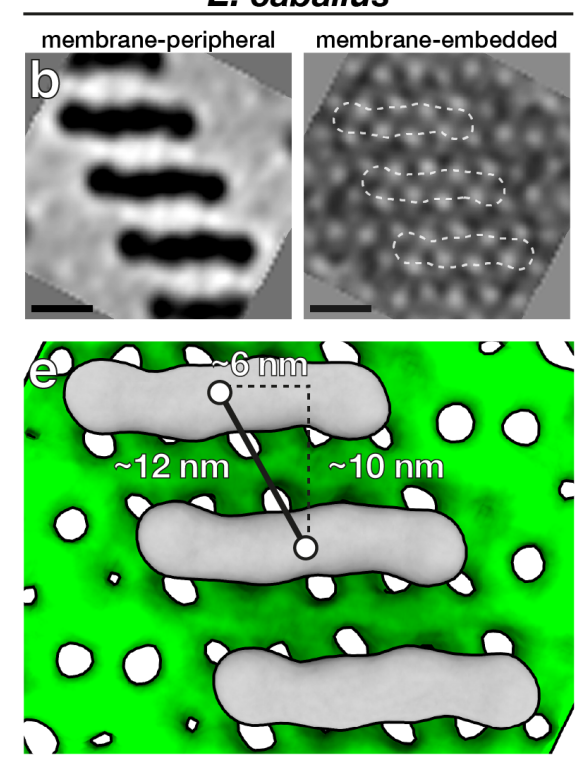

C $90^{\circ}$

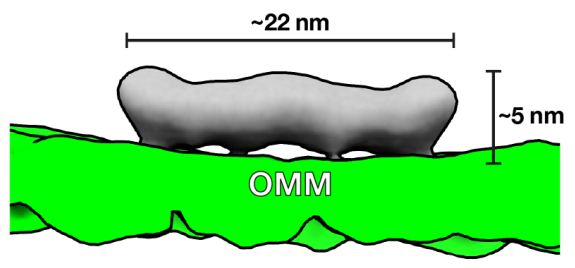

M. musculus
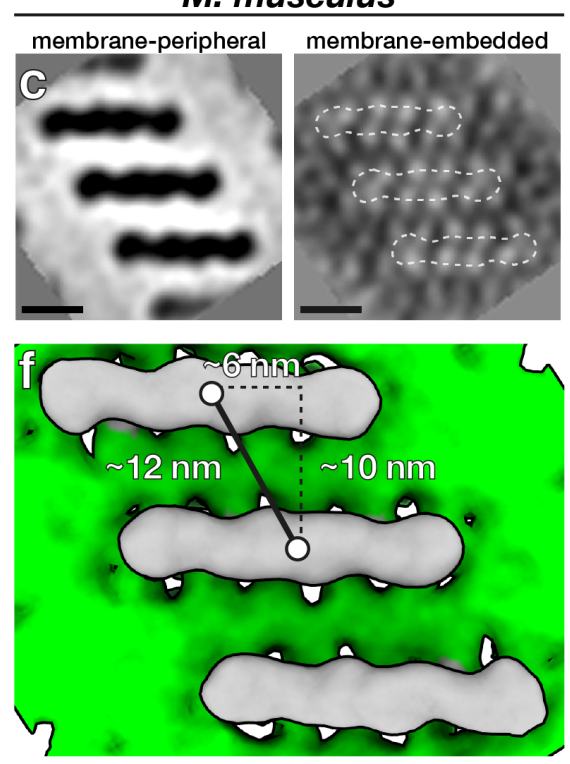

C $90^{\circ}$

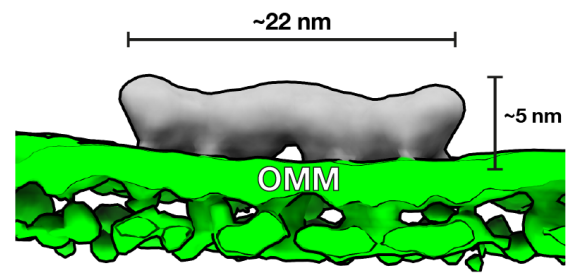

g
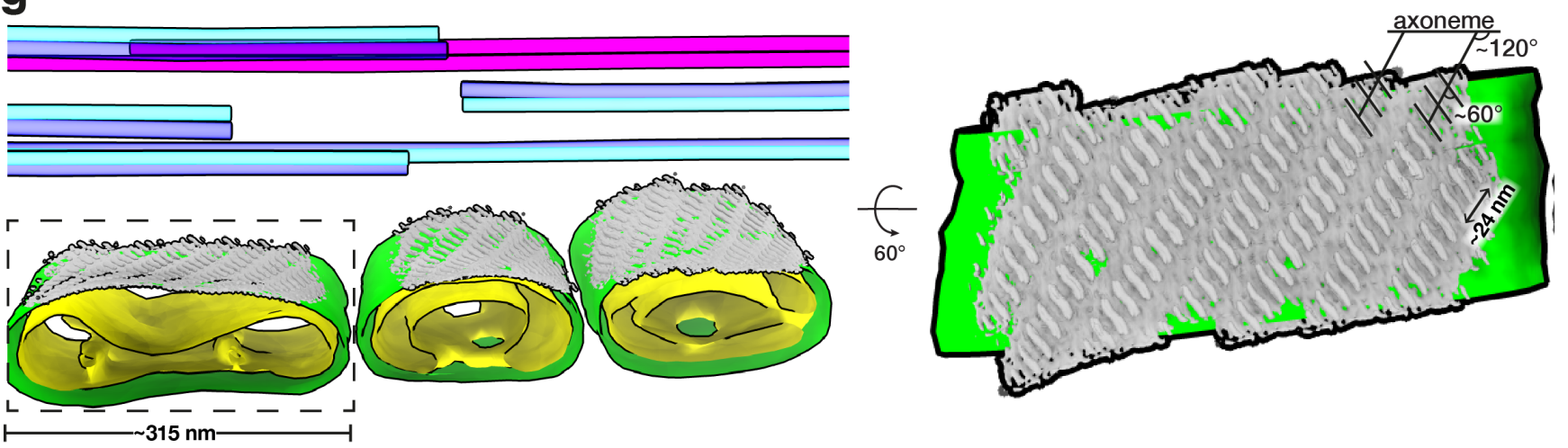

Fig. 3. Ordered protein arrays at the mitochondria-cytoskeleton interface are conserved across species. (a-c) Subtomogram averages of the protein arrays and underlying outer mitochondrial membrane (OMM) after applying twofold symmetry (note that density is black). (d-f) Isosurface renderings of the subtomogram averages in (a-c) with boat-shaped particles in grey and the OMM in green. (g) Left panel: Segmentation of the tomogram shown in Figure 2a, with the OMM in green, the IMM in yellow, microtubule doublets in blue, and the cpa in pink. Subtomogram averages of boat-shaped particles are colored grey and plotted back into their positions and orientations in the tomogram. Right panel: Rotated and zoomed-in view of the axoneme-facing surface of a mitochondrion. The axoneme is oriented horizontally, so the ladder-like arrays are oriented $\sim 120^{\circ}$ to the flagellar long axis, and individual particles within the array are oriented $\sim 60^{\circ}$ to this axis. Scale bars: (a-c) $10 \mathrm{~nm}$.

OMM (Gonçalves et al., 2007; Guo and Mannella, 1993; 277 Hoogenboom et al., 2007; Mannella, 1982). Indeed, our 278 label-free quantitative proteomics experiments show that the 279 most abundant OMM proteins in pig sperm are VDAC2 and 280 VDAC3 (Table S2). Furthermore, the lattice dimensions in 281 our averages closely match those of VDAC in purified Neu- 282 rospora OMM (Guo and Mannella, 1993; Mannella, 1998). 283 The lattice can be modeled by fitting multiple copies of the
VDAC2 crystal structure (Schredelseker et al., 2014) (Fig. S4a). We oriented VDAC2 in the membrane plane based on its known topology (Bayrhuber et al., 2008; Tomasello et al., 2013); however, at the current resolution, we cannot determine the orientation around the pore axis. Thus, in our model, each boat-shaped particle stretches across 8 VDAC molecules (Fig. 3). 
bioRxiv preprint doi: https://doi.org/10.1101/2021.02.16.431372; this version posted February 17, 2021. The copyright holder for this preprint (which was not certified by peer review) is the author/funder, who has granted bioRxiv a license to display the preprint in perpetuity. It is made available under aCC-BY 4.0 International license.
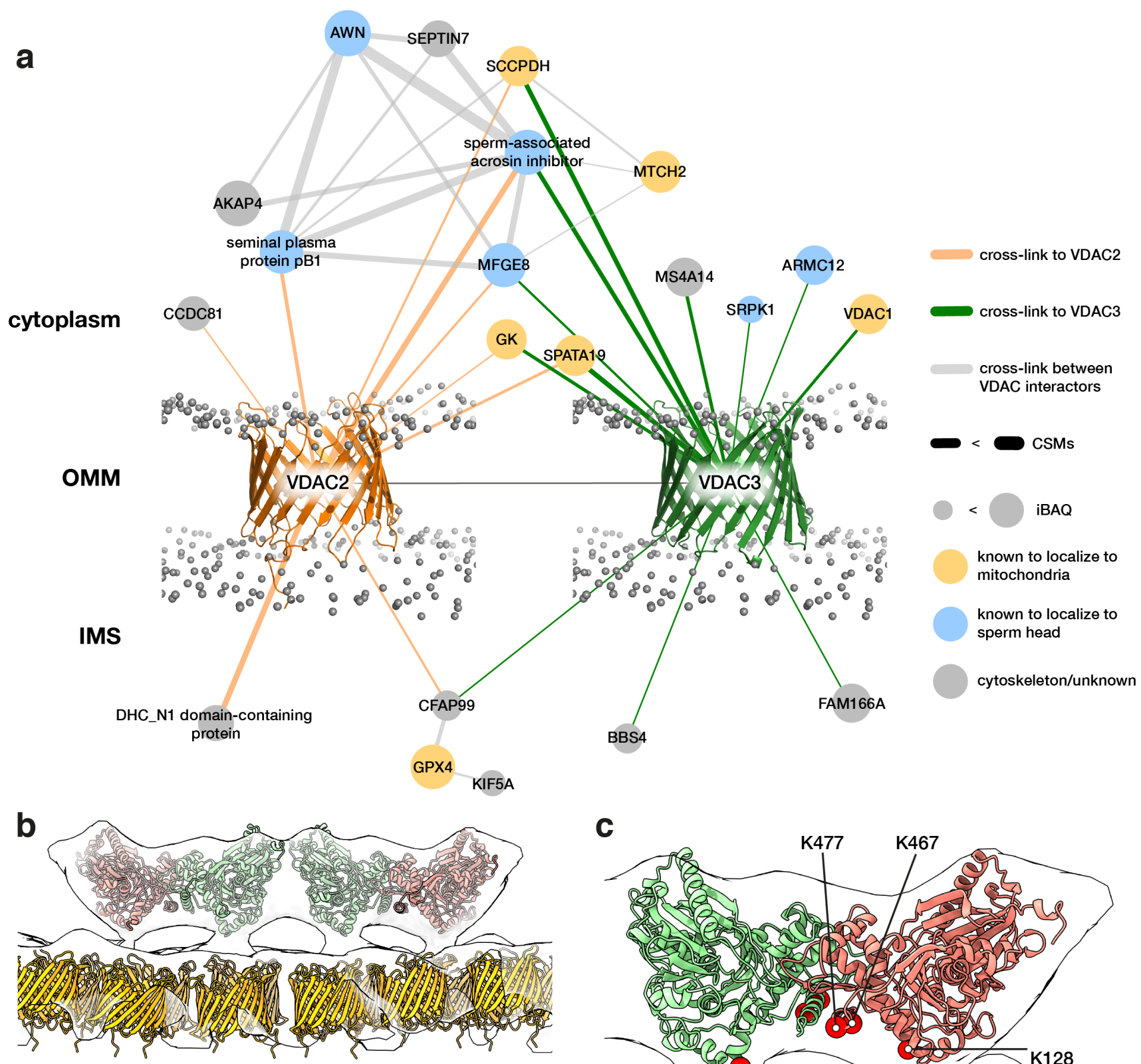

C

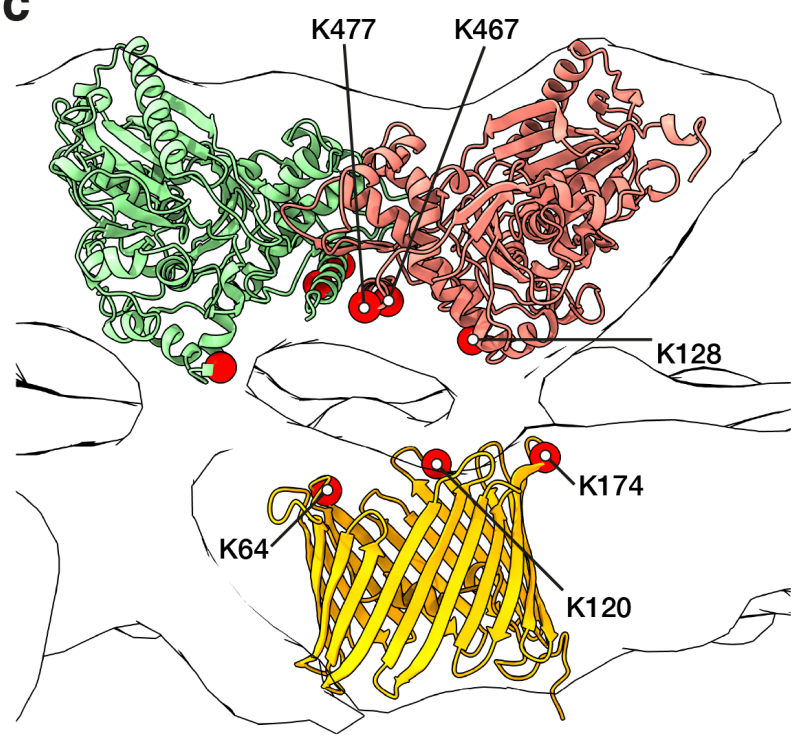

Fig. 4. Modelling the outer mitochondrial membrane (OMM) array as glycerol kinase-like (GK) proteins anchored on voltage dependent anion channels (VDACs). (a) The VDAC2/VDAC3 interactome derived from in-cell XL-MS of pig sperm. Protein nodes are colored according to their known subcellular localizations (yellow - mitochondria, blue - head, grey - cytoskeleton/unknown). Gray spheres indicate the phosphate groups of a simulated lipid bilayer which was structurally aligned based on the simulation for monomeric mouse VDAC1 (PDB 4C69) obtained from the MemProtMD server (Newport et al., 2019). (b) Modeling the OMM array as GK-like proteins anchored on VDACs. A GK-like dimer-of-dimers homology model (red and green) and VDAC homology models (yellow) were fitted into the pig subtomogram average map (white). (c) The positions of cross-linked Lys residues (red circles) are consistent with GK and VDAC orientation assignments in our model. 
Glycerol kinase-like proteins are probable con- 341 stituents of the conserved arrays at the mitochondria- 342 cytoskeleton interface. To search for possible constituents 343 of the protein arrays on the VDAC lattice, we used in-cell 344 cross-linking mass spectrometry (XL-MS) (Fasci et al., 2018; 345 Liu et al., 2018) to find potential VDAC2/VDAC3 interac- 346 tion partners on the OMM (Fig. 4). We treated pig sperm ${ }_{347}$ cells with the cross-linker disuccinimidyl sulfoxide (DSSO), ${ }_{348}$ which covalently links free lysines that are within $\sim 3 \mathrm{~nm}_{349}$ $(\mathrm{C} \alpha-\mathrm{C} \alpha)$ of each other. To increase confidence, we screened 350 for cross-links identified with at least two cross-link spectral matches (CSMs) (see Materials and Methods for details).

We first screened candidate proteins based on their known subcellular localizations (Fig. 4a). VDAC2/VDAC3 cross- $^{352}$ linked to mitochondria-associated proteins as well as to ${ }^{353}$ sperm head-associated proteins. This is consistent with im- ${ }^{354}$ munofluorescence studies localizing VDAC2/VDAC 3 both to ${ }^{355}$ the midpiece and to the acrosome, a large vesicle capping ${ }^{35}$ the anterior sperm nucleus (Hinsch et al., 2004; Kwon et al., 2013). Of the proteins in the mitochondria-associated inter- ${ }^{3}$ action hub, three proteins are particularly noteworthy because ${ }^{359}$ they are known to localize to the OMM and because their ${ }^{360}$ disruption results in dysplasia of the mitochondrial sheath: ${ }^{36}$ spermatogenesis-associated protein 19 (SPATA19) (Mi et al., ${ }^{36}$ 2015), glutathione peroxidase 4 (GPX4) (Imai et al., 2009; ${ }^{36}$ Schneider et al., 2009), and glycerol kinase (GK) (Chen et ${ }^{36}$ al., 2017b; Shimada et al., 2019).

To distinguish among these candidates, we compared the ${ }_{367}^{366}$ location of the cross-links with the known topology of VDAC ${ }_{368}$ in the OMM (Bayrhuber et al., 2008; Tomasello et al., 2013). ${ }_{369}$ GPX4 would interact on the side facing the intermembrane ${ }_{370}$ space, whereas SPATA19 and GK would interact on the cy- ${ }_{371}$ toplasmic face. Both SPATA19 and GK are highly abundant ${ }_{372}$ (Table S2), as would be expected for proteins forming exten- ${ }_{373}$ sive arrays. Assuming an average protein density of $\sim 1.43^{374}$ $\mathrm{g} / \mathrm{cm}^{3}$ (Quillin and Matthews, 2000), which corresponds to ${ }_{375}$ $\sim 0.861 \mathrm{Da} / \AA^{3}$, we estimate that each boat-shaped particle in ${ }_{376}$ the array has a molecular weight of $\sim 250 \mathrm{kDa}$. SPATA19 is 377 a small protein with an estimated molecular weight of $\sim 18$ $\mathrm{kDa}$. To fit into our EM densities, it must either be present ${ }_{379}$ in multiple copies or form a complex with other proteins. In $_{380}$ contrast, GK has an estimated molecular weight of $\sim 60 \mathrm{kDa}_{381}$ and is known to form S-shaped dimers $(\sim 120 \mathrm{kDa})$ that are ${ }_{382}$ conserved from bacteria (Bystrom et al., 1999; Fukuda et ${ }_{383}$ al., 2016) to eukaryotes (Balogun et al., 2019; Schnick et al., ${ }_{384}$ 2009).

To build a GK-VDAC model based on our subtomogram 386 average, we used rigid-body fitting to place two GK dimers 387 end-to-end into a boat-shaped density (Fig. S4b, Fig. 4b). 388 These fits defined a clear orientation for GK, with the N- 389 termini pointing upwards and the C-terminal helices facing 390 the OMM (Fig. 4b). To validate our fits, we mapped the 391 cross-linked lysines onto the resulting model (Fig. 4c). All 392 cross-links were between the cytosolic face of VDAC2 and 393 the OMM-facing surface of GK, which is consistent with ${ }_{394}$ the orientation expected from our fits. Assigning GK-like 395 proteins as constituents of the ordered OMM arrays at the 396 mitochondria-cytoskeleton interface is also supported by recent genetic studies. Sperm from mice lacking sperm-specific GK isoforms, which do not show glycerol kinase activity in vitro (Pan et al., 1999), have disorganized mitochondrial sheaths (Chen et al., 2017b; Shimada et al., 2019). In these mice, spherical mitochondria properly align along the flagellum but fail to properly elongate and coil around the ODFs (Shimada et al., 2019). This phenotype is consistent with our data showing direct links between GK protein arrays and the submitochondrial reticulum (Fig. 2b-c).

\section{Discussion}

In this study, we used cryo-FIB milling-enabled cryoET to image the sperm mitochondrial sheath in three mammalian species. Our data reveal that overall mitochondrial dimensions are remarkably consistent in sperm from the same species (Fig. 1, S1). This contrasts with other mitochondriarich tissues such as skeletal muscle, where there are massive variations in mitochondrial size and morphology within individual cells (Vincent et al., 2019). In addition, we did not observe mitochondrial nanotunnels in any of the species we examined, in contrast to their relative abundance in muscle tissue (Vincent et al., 2017, 2019). Our data also show that mitochondrial dimensions and cristae architecture vary across species (Fig. 1), providing possible structural bases for interspecific differences in mitochondrial energetics. Further comparative studies of how mitochondrial structure varies with sperm metabolism will undoubtedly contribute to our broader understanding of how mitochondrial form relates to function.

Our data show that, despite this diversity, the molecular underpinnings of mitochondrial sheath architecture are conserved, at least in mammals. Specifically, we identified novel inter-mitochondrial linkers that tether adjacent mitochondria (Fig. 1, S2) and arrays of boat-shaped particles that anchor mitochondria to the cytoskeleton (Fig. 2,3). In-cell subtomogram averaging and in-cell XL-MS suggest that these arrays consist of GK-like proteins anchored on VDAC lattices in the OMM (Fig. 4). Given that VDACs are ubiquitous OMM proteins, our findings motivate further efforts to explore whether they also regulate mitochondria-cytoskeleton interactions in other cell types.

The OMM arrays may function to regulate the precise elongation and coiling of mitochondria, contributing to the striking consistency within the mitochondrial sheath. In mature sperm, these arrays may help maintain the integrity of mitochondria-cytoskeleton contacts, stabilizing them against shear stresses during sperm motility and hyperactivation. However, it is unclear what determines the organization of these arrays in the first place. Our averages do not hint at direct interactions between boat-shaped particles. Instead, their spacing may be defined by the organization of the underlying VDAC lattice. Another intriguing possibility is that the arrays are organized by their cytoskeletal binding partners; the periodicity of relevant motifs on the submitochondrial reticulum could dictate the spacing of the OMM arrays.

To our knowledge, this is the first time such assemblies 
have been visualized at any organelle-cytoskeleton interface ${ }_{448}$ in any cell type. Defining whether similar arrays are present 449 in other differentiated cell types - and whether they use a sim- 450 ilar pool of protein components - is an area ripe for study. In 451 striated muscle, proper mitochondrial positioning is critical ${ }_{452}$ for muscle function and depends on direct associations be- 453 tween mitochondria and intermediate filaments (Konieczny 454 et al., 2008; Milner et al., 2000). Similarly, in skin cells, 455 mitochondrial organization depends on keratin (Steen et al., 456 2020). The structural bases for these associations are un- ${ }_{457}$ known, but cryo-ET and in-cell XL-MS may prove useful in ${ }_{458}^{457}$ these contexts as well.

\section{Acknowledgements}

The authors thank Dr. M Vanevic for excellent compu- ${ }^{462}$ tational support; Dr. D Vasishtan for providing scripts that ${ }^{463}$ greatly facilitated subtomogram averaging; Ingr. CTWM ${ }^{464}$ Schneijdenberg and JD Meeldijk for managing and maintain- ${ }^{465}$ ing the Utrecht University EM Square facility; Stal Schep ${ }^{466}$ (Tull en het Waal, The Netherlands) for providing horse se- ${ }^{467}$ men; MW Haaker and M Houweling for providing mouse ${ }^{468}$ reproductive tracts; Prof. F Förster and Prof. A Akhmanova 469 for critical reading of the manuscript; and Prof. EY Jones for 470 insightful discussions. The authors also thank the Henriques 471 Lab for the publicly-available $\mathrm{LTT}_{\mathrm{E}} \mathrm{X}$ template. This work 472 benefitted from access to the Netherlands Center for Electron 473 Nanoscopy (NeCEN) with support from operators Dr. RS 474 Dillard and Dr. CA Diebolder and IT support from B Alewi- 475 jnse. RZC, JFH, and AJRH acknowledge support from NWO 476 funding the Netherlands Proteomics Centre through the X-477 omics Road Map program (project 184.034.019). This work 478 was funded by NWO Start-Up Grant 740.018.007 to TZ, and 479 MRL is supported by a Clarendon Fund-Nuffield Department 480 of Medicine Prize Studentship.

\section{Author Contributions}

PM, MZ, HH, EGB, and BMG provided sperm samples. ${ }_{485}$ MRL, MCR, and RTR prepared samples for cryo-ET. MRL ${ }_{486}$ performed cryo-FIB milling. MRL, MCR, RTR, SCH, and ${ }_{487}$ TZ collected cryo-ET data. MRL and MCR processed cryo- ${ }_{488}$ ET data. MRL, MCR, and TZ analyzed cryo-ET data. RZC ${ }_{489}$ and JFH performed all proteomics and cross-linking mass spectrometry experiments along with corresponding structural modelling under the supervision of AJRH. MRL and TZ wrote the manuscript, and all authors contributed to revi- ${ }^{490}$ sions.

\section{Declaration of Interests}

The authors declare no competing interests.

\section{Materials and Methods}

Sperm collection and preparation. Pig sperm samples 499 were purchased from an artificial insemination company 500 (Varkens KI Nederland), stored at $18^{\circ} \mathrm{C}$, and prepared for 501 imaging within 1 day of delivery. Sperm were layered onto a 502 discontinuous gradient consisting of $4 \mathrm{~mL}$ of $35 \%$ Percoll® (GE Healthcare) underlaid with $2 \mathrm{~mL}$ of $70 \%$ Percoll ${ }^{\circledR}$, both in HEPES-buffered saline (HBS: $20 \mathrm{mM}$ HEPES, $137 \mathrm{mM}$ $\mathrm{NaCl}, 10 \mathrm{mM}$ glucose, $2.5 \mathrm{mM} \mathrm{KCl}, 0.1 \%$ kanamycin, $\mathrm{pH}$ 7.6) and centrifuged at $750 \mathrm{~g}$ for $15 \mathrm{~min}$ at RT (Harrison et al., 1993). Pelleted cells were washed once in phosphatebuffered saline (PBS: $137 \mathrm{mM} \mathrm{NaCl}, 3 \mathrm{mM} \mathrm{KCl}, 8 \mathrm{mM}$ $\mathrm{Na}_{2} \mathrm{HPO}_{4}, 1.5 \mathrm{mM} \mathrm{KH} \mathrm{PO}_{4}, \mathrm{pH}$ 7.4), resuspended in PBS and counted with a hemocytometer.

Horse semen was collected from mature Warmblood stallions using a Hanover artificial vagina in the presence of a teaser mare. After collection, semen was filtered through gauze to remove gel fraction and large debris, then transported to the laboratory at $37^{\circ} \mathrm{C}$ and kept at room temperature until further processing. Semen was diluted in INRA96 (IMV Technologies) to obtain a sperm concentration of 30 x $10^{6}$ cells $/ \mathrm{mL}$. After this, sperm were centrifuged through a discontinuous Percoll gradient as described above for pig sperm for $10 \mathrm{~min}$ at $300 \mathrm{~g}$ followed by $10 \mathrm{~min}$ at $750 \mathrm{~g}$ (Harrison et al., 1993). The remaining pellet was resuspended in $1 \mathrm{~mL}$ of PBS and centrifuged again for $5 \mathrm{~min}$ at $750 \mathrm{~g}$.

Mouse sperm were collected from the cauda epididymis of adult male C75BL/6 mice as described in (Hutcheon et al., 2017). Briefly, male mice were culled as described in (Mederacke et al., 2015) and the cauda epididymides were dissected with the vas deferens attached and placed in a $500 \mu \mathrm{L}$ droplet of modified Biggers, Whitten, and Whittingham media (BWW: $20 \mathrm{mM}$ HEPES, $91.5 \mathrm{mM} \mathrm{NaCl}, 4.6$ $\mathrm{mM} \mathrm{KCl}, 1.7 \mathrm{mM}$ D-glucose, $0.27 \mathrm{mM}$ sodium pyruvate, $44 \mathrm{mM}$ sodium lactate, $5 \mathrm{U} / \mathrm{mL}$ penicillin, and $5 \mu \mathrm{g} / \mathrm{mL}$ streptomycin, adjusted to $\mathrm{pH} 7.4$ and an osmolarity of 300 $\mathrm{mOsm} / \mathrm{kg}$ ). To retrieve the mature cauda spermatozoa from the epididymides, forceps were used to first gently push the stored sperm from the vas deferens, after which two incisions were made with a razor blade in the cauda. Spermatozoa were allowed to swim out of the cauda into the BWW over a period of $15 \mathrm{~min}$ at $37^{\circ} \mathrm{C}$, after which the tissue was removed and sperm were loaded onto a $27 \%$ Percoll density gradient and washed by centrifugation at $400 \mathrm{~g}$ for $15 \mathrm{~min}$. The pellet consisting of an enriched sperm population was resuspended in BWW and again centrifuged at $400 \mathrm{~g}$ for 2 min to remove excess Percoll.

Cryo-EM grid preparation. Typically, $3 \mu \mathrm{L}$ of a suspension containing either $2-3 \times 10^{6}$ cells $/ \mathrm{mL}$ (for whole cell tomography) or 20-30 x $10^{6}$ cells $/ \mathrm{mL}$ (for cryo-FIB milling) was pipetted onto a glow-discharged Quantifoil R 2/1 200mesh holey carbon grid. One $\mu \mathrm{L}$ of a suspension of BSAconjugated gold beads (Aurion) was added, and the grids then blotted manually from the back (opposite the side of cell deposition) for $\sim 3 \mathrm{~s}$ (for whole cell tomography) or for $\sim 5-6 \mathrm{~s}$ (for cryo-FIB milling) using a manual plunge-freezer (MPI Martinsreid). Grids were immediately plunged into a liquid ethane-propane mix (37\% ethane) (Tivol et al., 2008) cooled to liquid nitrogen temperature. Grids were stored under liquid nitrogen until imaging. 
Cryo-focused ion beam milling. Grids were mounted into 557 modified Autogrids (ThermoFisher) for mechanical support. 558 Clipped grids were loaded into an Aquilos (ThermoFisher) 559 dual-beam cryo-focused ion beam/scanning electron micro- 560 scope (cryo-FIB/SEM). All SEM imaging was performed at 561 $2 \mathrm{kV}$ and $13 \mathrm{pA}$, whereas FIB imaging for targeting was per- 562 formed at $30 \mathrm{kV}$ and $10 \mathrm{pA}$. Milling was typically performed 563 with a stage tilt of $18^{\circ}$, so lamellae were inclined $11^{\circ}$ rela- 564 tive to the grid. Each lamella was milled in four stages: an 565 initial rough mill at $1 \mathrm{nA}$ beam current, an intermediate mill 566 at $300 \mathrm{pA}$, a fine mill at $100 \mathrm{pA}$, and a polishing step at $30_{567}$ pA. Lamellae were milled with the wedge pre-milling tech- 568 nique described in (Schaffer et al., 2017) and with expansion 569 segments as described in (Wolff et al., 2019).

Tilt series acquisition. Tilt series were acquired on ei- 572 ther a Talos Arctica (ThermoFisher) operating at $200 \mathrm{kV}_{573}$ or a Titan Krios (ThermoFisher) operating at $300 \mathrm{kV}$, both ${ }_{574}$ equipped with a post-column energy filter (Gatan) in zero- 575 loss imaging mode with a $20-\mathrm{eV}$ energy-selecting slit. All 576 images were recorded on a K2 Summit direct electron detec- 577 tor (Gatan) in either counting or super-resolution mode with 578 dose-fractionation. Tilt series were collected using SerialEM ${ }_{579}$ (Mastronarde, 2005) at a target defocus of between -4 and -6580 $\mu \mathrm{m}$ (conventional defocus-contrast) or between -0.5 and $-1.5{ }_{581}$ $\mu \mathrm{m}$ (for tilt series acquired with the Volta phase plate). Tilt ${ }_{582}$ series were typically recorded using either strict or grouped ${ }_{583}$ dose-symmetric schemes, either spanning $\pm 56^{\circ}$ in $2^{\circ}$ incre- ${ }_{584}$ ments or $\pm 54^{\circ}$ in $3^{\circ}$ increments, with total dose limited to 585 $\sim 100 e^{-} / \AA^{2}$.

Tomogram reconstruction. Frames were aligned either ${ }_{588}$ post-acquisition using Motioncor2 1.2.1 (Zheng et al., 2017) or on-the-fly using Warp (Tegunov and Cramer, 2019). 589 Frames were usually collected in counting mode; when 590 super-resolution frames were used, they were binned $2 \mathrm{X}$ dur- 591 ing motion correction. Tomograms were reconstructed in 592 IMOD (Kremer et al., 1996) using weighted back-projection, 593 with a SIRT-like filter (Zeng, 2012) applied for visualization 594 and segmentation. Defocus-contrast tomograms were CTF- 595 corrected in IMOD using ctfphaseflip while VPP tomograms 596 were left uncorrected.

Tomogram segmentation. Segmentation was generally 599 performed semi-automatically using the neural network- 600 based workflow implemented in the TomoSeg package in 601 EMAN 2.21 (Chen et al., 2017). Microtubules, however, 602 were traced manually in IMOD. Segmentation was then man- 603 ually refined in Chimera 1.12 (Pettersen et al., 2004) or in ${ }_{604}$ ChimeraX (Goddard et al., 2018). Visualization was per- 605 formed in ChimeraX.

Subtomogram averaging of ATP synthase and outer ${ }_{608}$ mitochondrial membrane arrays. Subtomogram averag- 609 ing with missing wedge compensation was performed using ${ }_{610}$ PEET 1.13.0 (Heumann et al., 2011; Nicastro et al., 2006).

Resolution was estimated using the Fourier shell correlation 611 (FSC) at a cut-off of 0.5 (Nicastro et al., 2006). Alignments 612 were generally performed first on binned data, after which aligned positions and orientations were transferred to lessbinned data using scripts generously provided by Dr. Daven Vasishtan. Details of acquisition parameters and particle numbers are summarized in Table S1.

Alignment strategies for these complexes were designed to take advantage of their defined orientations relative to the membrane plane. Particles were picked manually and their initial orientations were defined using stalkInit. Initial references were either a randomly chosen particle (for ladder-like arrays) or an average of all particles after roughly aligning them based on their initial orientations (for ATP synthase). Independent alignments using independent initial references were performed for datasets from different species. Alignments allowed for large rotational search ranges around the particle long axis (defined as the y-axis, perpendicular to the membrane plane), with limited search ranges around the $\mathrm{x}$ and z-axes (the membrane plane).

All initial alignments were performed without symmetry. After visual inspection of the maps, twofold symmetry was applied for ladder-like arrays. Symmetrization involved using the aligned positions from the unsymmetrized runs as seed points and rotating particles around the axis of symmetry to generate virtual particles. A symmetrized volume was generated by averaging all particles and virtual particles and used as a reference for a final, restricted alignment.

Plotbacks were generated in IMOD by first running createAlignedModel to generate model files reflecting updated particle positions and orientations after alignment. The relevant subtomogram average was then thresholded for visualization and saved as an isosurface model, which was then placed back into the tomograms using clonemodel.

Measurements and quantification. All measurements of mitochondrial width were performed in IMOD on Volta phase plate tomograms filtered with a SIRT-like filter. Mitochondrial width was measured in the non-missing wedge direction at five points along the length of each mitochondrion. Only mitochondria that were entirely in the field of view were included in the measurements. Tomograms and corresponding measurements were then grouped based on their locations relative to the connecting piece, which were determined based on low-magnification images used for targeting during data acquisition.

Internal mitochondrial ultrastructure was quantified from tomograms from cryo-FIB milled lamellae. The volume occupied by the matrix $\left(\mathrm{V}_{\text {matrix }}\right.$, the volume enclosed by the IMM) was measured relative to the volume occupied by the entire mitochondrion $\left(\mathrm{V}_{\text {mito }}\right.$, the volume enclosed by the OMM). Mesh volumes were extracted from segmentations using imodinfo. Because neural network-based segmentation often resulted in gaps, mitochondrial membranes were segmented manually in IMOD for quantification. Only slices in which both the IMM and OMM were clearly defined were used for segmentation.

Cross-linking, lysis, digestion and peptide fractionation. All proteomics and cross-linking mass spectrometry ex- 
periments were performed on Percoll-washed pig sperm pre- 670 pared as described above. For cross-linking, approximately 671 300 x 106 cells were used from 3 different animals. Briefly, 672 pelleted sperm cells were resuspended in $540 \mu \mathrm{L}$ of $\mathrm{PBS}_{673}$ supplemented with disuccinimidyl sulfoxide (DSSO, Thermo 674 Fisher Scientific) to a final concentration of $1 \mathrm{mM}$. The reac- 675 tion mix was incubated for $30 \mathrm{~min}$ at $25^{\circ} \mathrm{C}$ with $700 \mathrm{rpm} 676$ shaking in a ThemoMixer $\mathrm{C}$ (Eppendorf) and subsequently 677 quenched for 20 min by adding Tris- $\mathrm{HCl}$ (final concentration $50 \mathrm{mM}$ ). Cross-linked cells were spun down at $13800 \mathrm{~g} 678$ for $10 \mathrm{~min}$ at $4^{\circ} \mathrm{C}$, after which the supernatant was removed. 679 Cells were then lysed according to a protocol modified from 680 (Potel et al., 2018). Cells were resuspended in $1 \mathrm{~mL}$ of ly- 681 sis buffer $(100 \mathrm{mM}$ Tris- $\mathrm{HCl} \mathrm{pH} 8.5,7 \mathrm{M}$ Urea, $1 \%$ Triton 682 $\mathrm{X}-100,5 \mathrm{mM}$ TCEP, $30 \mathrm{mM}$ CAA, $10 \mathrm{U} / \mathrm{ml}$ DNase I, $1 \mathrm{mM}$ $\mathrm{MgCl} 2,1 \%$ benzonase (Merck Millipore, Darmstadt, Ger- ${ }_{684}$ many), $1 \mathrm{mM}$ sodium orthovanadate, phosphoSTOP phos- 685 phatases inhibitors, and cOmpleteTM Mini EDTA-free pro- 686 tease inhibitors). Cells were sonicated on ice for 2 min us- 687 ing an ultrasonic processor (UP100H, Hielscher) at $80 \%$ am- 688 plitude. The proteins were then precipitated according to 689 (Wessel and Flügge, 1984) and the dried protein pellet re- 690 suspended in digestion buffer (100 mM Tris-HCl pH 8.5, $1 \% 691$ sodium deoxycholate (Sigma-Aldrich), $5 \mathrm{mM}$ TCEP, and 30692 mM CAA). Trypsin and Lys-C proteases were added to a 1:25 693 and 1:100 ratio $(\mathrm{w} / \mathrm{w})$ respectively and protein digestion per- 694 formed overnight at $37^{\circ} \mathrm{C}$. The final peptide mixtures were 695 desalted with solid-phase extraction C18 columns (Sep-Pak, 696 Waters) and fractionated with an Agilent 1200 HPLC pump ${ }_{697}$ system (Agilent) coupled to a strong cation exchange (SCX) 698 separation column (Luna SCX $5 \mu \mathrm{m}-100 \AA$ particles, 50 x 699 $2 \mathrm{~mm}$, Phenomenex), resulting in 25 fractions.

\section{Liquid chromatography with mass spectrometry. Ap- 701} proximately $1000 \mathrm{ng}$ of peptides from each biological repli- 702 cate before SCX fractionation were first injected onto an Ag- 703 ilent 1290 Infinity UHPLC system (Agilent) on a 50-cm an- 704 alytical column packed with $\mathrm{C} 18$ beads (Dr Maisch Reprosil ${ }_{705}$ C18, $3 \mu \mathrm{m}$ ) coupled online to an Orbitrap HF-X (Thermo ${ }_{706}$ Fisher Scientific). For this classical bottom-up analysis, we ${ }_{707}$ used the following LC-MS/MS parameters: after 5 min of ${ }_{708}$ loading with $100 \%$ buffer A (H2O with $0.1 \%$ formic acid), ${ }_{709}$ peptides were eluted at $300 \mathrm{~nL} / \mathrm{min}$ with a 95-min gradient from $13 \%$ to $40 \%$ of buffer B (80\% acetonitrile and $20 \% 710$ $\mathrm{H} 2 \mathrm{O}$ with $0.1 \%$ formic acid). For MS acquisition we used an 711 MS1 Orbitrap scan at 60,000 resolution, automatic gain con- 712 trol (AGC) target of $3 \times 106$ ions and maximum inject time ${ }_{713}$ of $20 \mathrm{~ms}$ from 375 to $1600 \mathrm{~m} / \mathrm{z}$; the 15 most intense ions 714 were submitted to MS2 Orbitrap scan at 30,000 resolution, 715 AGC target of 1 x 105 ions and maximum inject time of $50_{716}$ $\mathrm{ms}$ (isolation window of $1.4 \mathrm{~m} / \mathrm{z}, \mathrm{NCE}$ at $27 \%$ and dynamic exclusion of 16 seconds). The SCX fractions were analyzed with same Agilent HPLC and the same nano-column coupled on-line to an Orbitrap Lumos mass spectrometer (ThermoFisher Scientific). For these runs, we used a gradient from $6 \%$ to $39 \%$ buffer B over 100 min with specific MS settings for DSSO cross-links: survey MS1 Orbitrap scan at 60,000 resolution from 375 to 1500 , AGC target of 4 x 105 ions and maximum inject time of $50 \mathrm{~ms}$; MS2 Orbitrap scan at 30,000 resolution, AGC target of $5 \times 104$ ions, and maximum inject time of $100 \mathrm{~ms}$ for detection of DSSO signature peaks (difference in mass of $37.972 \mathrm{Da}$ ). The four ions with this specific difference were analyzed with a MS3 Ion Trap scans at AGC target of $2 \times 104$ ions, maximum inject time of 150 $\mathrm{ms}$ for sequencing selected signature peaks (representing the individual peptides).

Mass spectrometry data processing. The 3 raw files obtained with classical bottom-up approach were analyzed with MaxQuant version 1.6.17 with all the automatic settings adding Deamidation (N) as dynamic modification against the Sus scrofa reference proteome (Uniprot version of 08/2020 with 49,795 entries). With this search, we were able to calculate intensity-based absolute quantification (iBAQ) values and created a smaller FASTA file to use for analysis of cross-linking experiments (table as Supplementary information). Raw files for cross-linked cells were analyzed with Proteome Discoverer software suite version 2.4.1.15 (ThermoFisher Scientific) with the incorporated XlinkX node for analysis of cross-linked peptides as described in (Klykov et al., 2018). Data were searched against the smaller FASTA created in house with "MS2_MS3 acquisition strategy". For the XlinkX search, we selected full tryptic digestion with 3 maximum missed cleavages, 10 ppm error for MS1, 20 ppm for MS2, and 0.5 Da for MS3 in Ion Trap. For modifications, we used static Carbamidomethyl (C) and dynamic Oxidation (M), Deamidation (N) and Met-loss (protein N-term). The crosslinked peptides were accepted with a minimum score of 40 , minimum score difference of 4 and maximum FDR rate set to $5 \%$; further standard settings were used.

Interactome analysis, homology modelling, and cross-link mapping. The interaction map for VDAC proteins was generated in R (Grant et al., 2006) using the igraph package (v 1.2.4.2). Only cross-links with at least two crosslink spectral matches (CSMs) were used for network generation. Homology models of GK and VDAC2 were generated in Robetta (Kim et al., 2004) and fitted into subtomogram average maps by rigid body fitting in Chimera X. Cross-links were mapped onto the resulting models using ChimeraX.

Data availability. Subtomogram average maps have been deposited to the Electron Microscopy Data Bank (EMDB) with the following accession numbers: EMDB-12354, 12355,12356 , and 12357 . The model of putative glycerol kinase-like proteins anchored on a VDAC array has been deposited to the Protein Data Bank (PDB) with the accession number PDB ID 7NIE. 
bioRxiv preprint doi: https://doi.org/10.1101/2021.02.16.431372; this version posted February 17, 2021. The copyright holder for this preprint (which was not certified by peer review) is the author/funder, who has granted bioRxiv a license to display the preprint in perpetuity. It is made available under aCC-BY 4.0 International license.
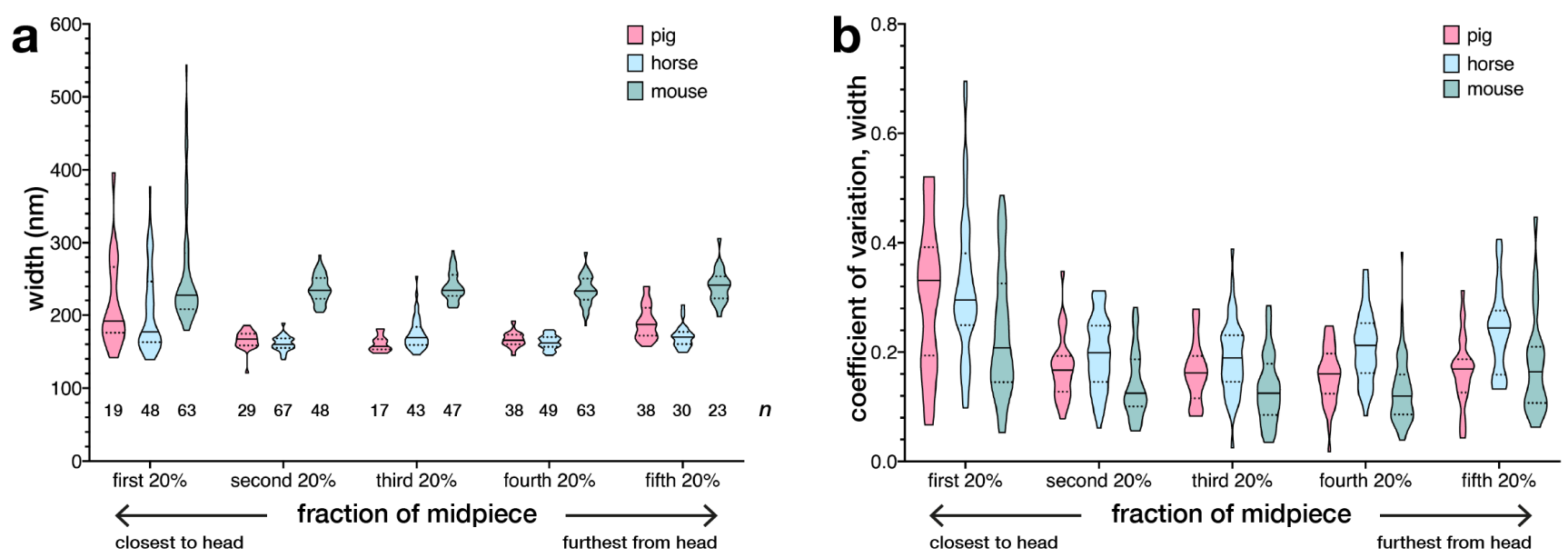

Fig. S1. Mitochondrial dimensions are consistent within species but vary across species and spatially along the midpiece. (a) Plotting the average width of mitochondria from different regions of the midpiece shows that mouse sperm mitochondria are larger than pig and horse sperm mitochondria. Note also that, in all three species, mitochondria at the proximal end of the midpiece are larger than those in more distal parts. (b) Mitochondrial width was measured at five points along the length of each mitochondrion. Plotting the coefficient of variation from different regions along the midpiece shows that mitochondria at the start of the midpiece have more variable widths along their lengths. In (a), $n$ indicates the number of mitochondria analyzed. In both (a) and (b), solid lines represent the median and dotted lines represent the first and third quartiles.

\section{S. scrofa}
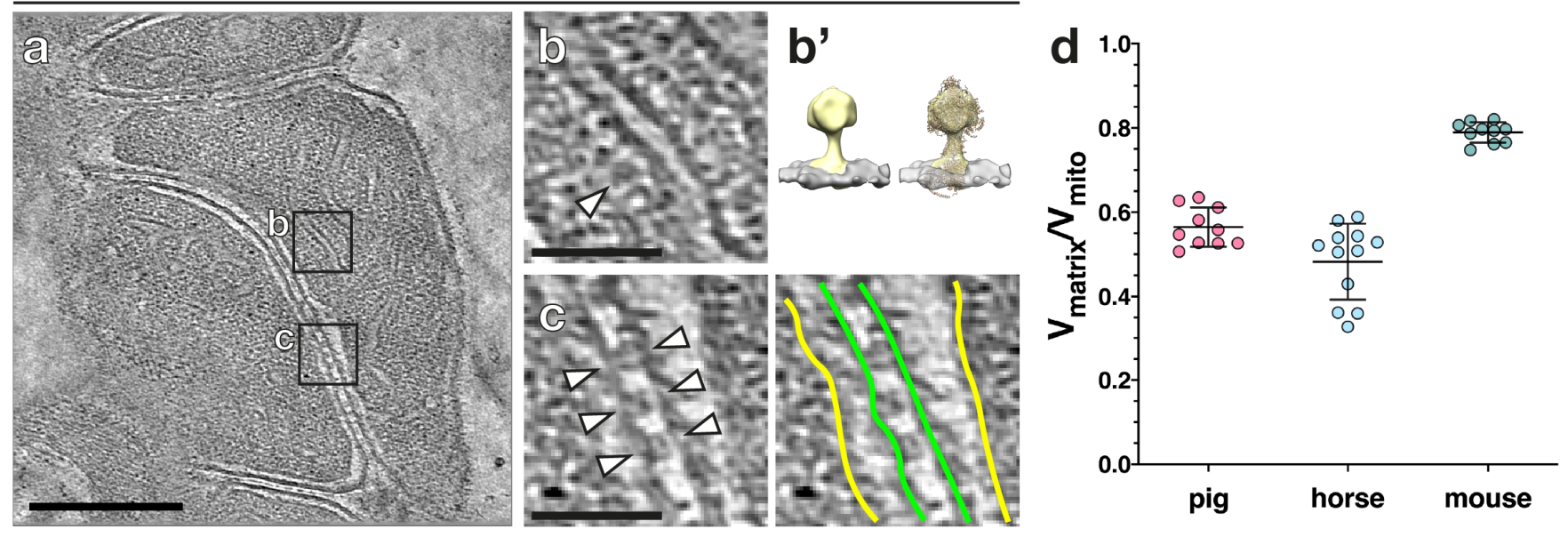

Fig. S2. Cryo-focused ion beam (cryo-FIB) milling reveals the internal organization of sperm mitochondria. (a) Slice through a cryo-tomogram of FIB-milled pig sperm mitochondria close to the connecting piece. (b) ATP synthase can be directly identified on cristae based on its characteristic shape, which is confirmed by subtomogram averaging (b'). (c) Novel inter-mitochondrial linkers tether neighboring mitochondria to each other (arrowheads in left panel). (d) Quantifying the volume enclosed by the mitochondrial matrix relative to the volume enclosed by the whole mitochondrion reveals that pig and horse sperm mitochondria have more expanded cristae and more condensed matrices than mouse sperm mitochondria. Lines represent mean \pm standard deviation.Scale bars: (a) $250 \mathrm{~nm}$, (b-c) $100 \mathrm{~nm}$. 
bioRxiv preprint doi: https://doi.org/10.1101/2021.02.16.431372; this version posted February 17, 2021. The copyright holder for this preprint (which was not certified by peer review) is the author/funder, who has granted bioRxiv a license to display the preprint in perpetuity. It is made available under aCC-BY 4.0 International license.
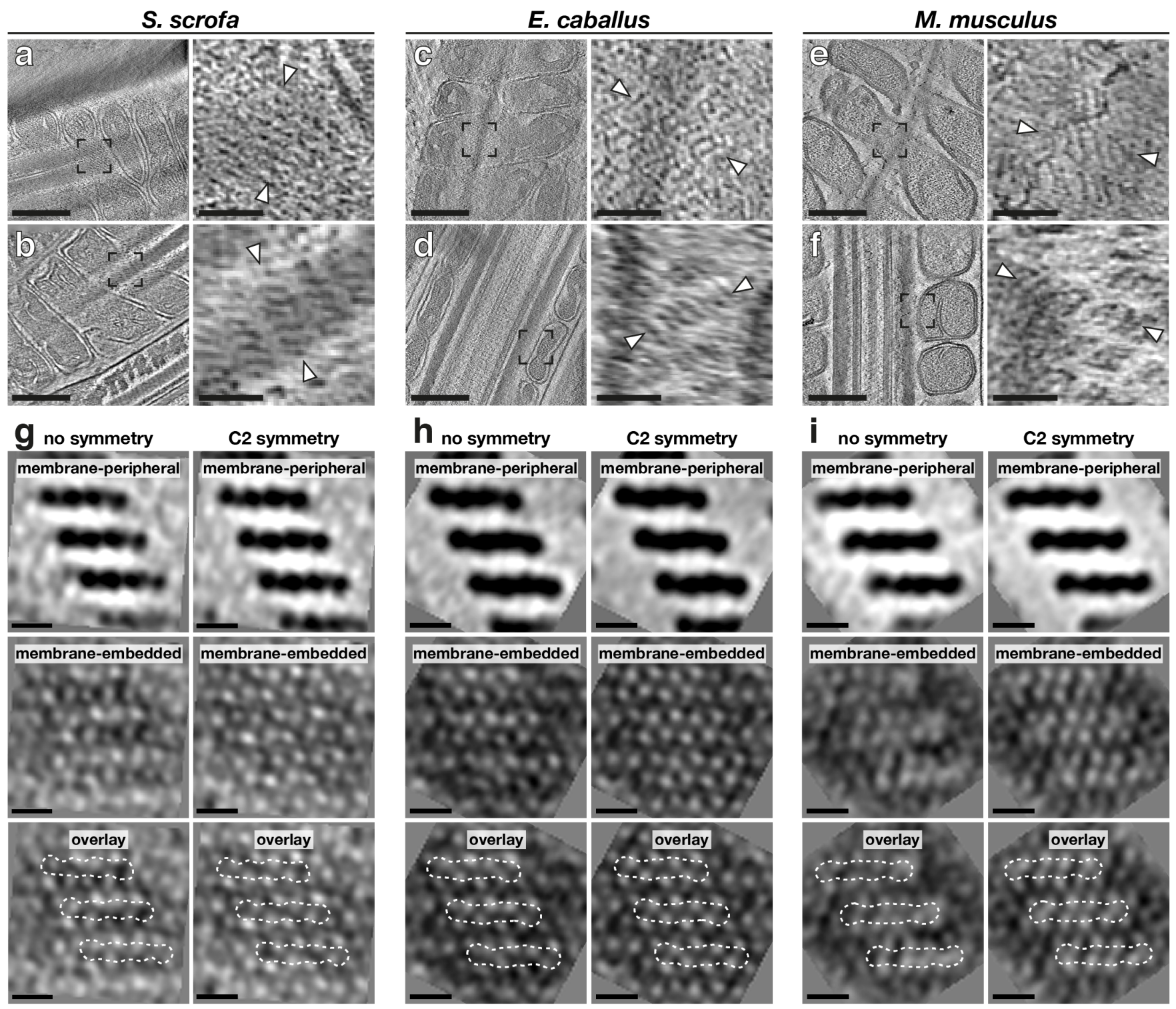

Fig. S3. The particles forming the ordered arrays at the mitochondria-cytoskeleton interface are two-fold symmetric. (a-f) Slices through cryo-tomograms of FIB-milled pig $(a, b)$, horse $(c, d)$, and mouse $(e, f)$ mitochondria. Right panels show digital zooms of the regions boxed out in the left panels, with arrowheads indicating arrays. (g-i) Subtomogram averages of the arrays and the outer mitochondrial membrane (OMM) without (left) and with (right) twofold symmetry. Scale bars: (a-f) $250 \mathrm{~nm}$, (g-i) $10 \mathrm{~nm}$. 
bioRxiv preprint doi: https://doi org/10.1101/2021.02 16.431372; this version posted February 17, 2021. The copyright holder for this preprint (which was not certified by peer review) is the author/funder, who has granted bioRxiv a license to display the preprint in perpetuity. It is made available under aCC-BY 4.0 International license.
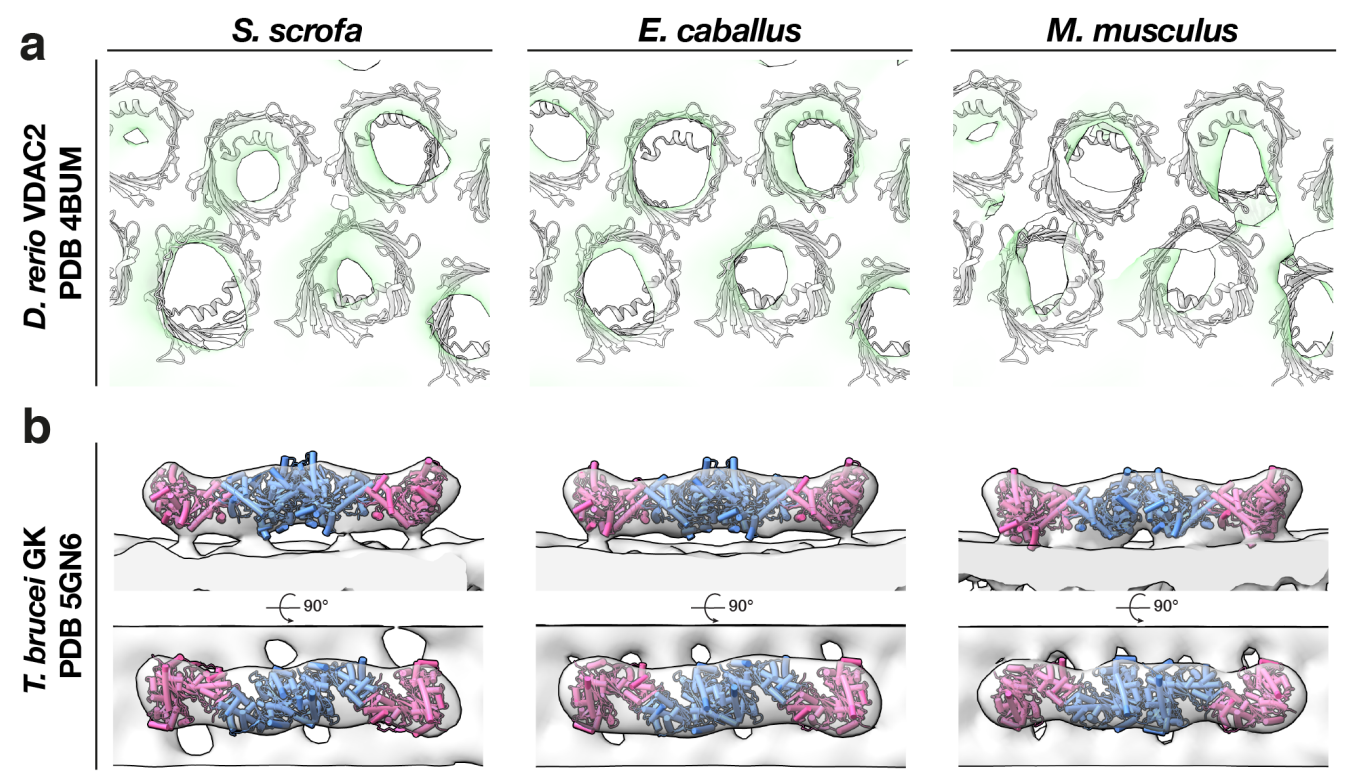

molmap @ $30 \AA$

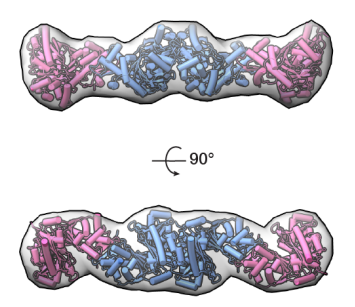

Fig. S4. Fitting crystal structures of glycerol kinase (GK and voltage dependent anion channels (VDACs) into the pig subtomogram average map. (a) The crystal structure of VDAC2 from zebrafish (PDB 4BUM) is shown in grey, fitted into the cryo-ET averaged map (green). (b) Two copies of a crystal structure of GK (pink and blue) from Trypanosoma brucei (PDB 5GN6) fitted into the cryo-ET averaged map (grey). On the right, the GK crystal structure is shown filtered to $30 \AA$ resolution. 
Table S1. Image acquisition and processing metrics for subtomogram averaging of mitochondrial protein complexes in mammalian sperm.

\begin{tabular}{llll}
\hline \multirow{2}{*}{ Parameter } & \multicolumn{3}{c}{ Species } \\
\cline { 2 - 4 } & Sus scrofa & Equus caballus & Mus musculus \\
\hline ATP synthase & & & \\
$\quad$ sample type & lamellae & - & - \\
$\quad$ number of cells used & 3 & - & - \\
microscope (accelerating voltage) & Arctica $(200 \mathrm{kV})$ & - & - \\
pixel size $(\AA)$ & 4.34 & - & - \\
symmetry & $\mathrm{C} 1$ & - & - \\
number of particles & 209 & - & - \\
estimated resolution $(\AA)$ & 38 & - & - \\
& & & \\
outer mitochondrial membrane arrays & & lamellae & lamellae \\
sample type & 3 & 3 & 8 \\
number of cells used & Arctica $(200 \mathrm{kV})$ & Arctica $(200 \mathrm{kV})$ & Arctica $(200 \mathrm{kV})$ \\
microscope (accelerating voltage) & 4.34 & 5.66 & 5.66 \\
pixel size $(\AA)$ & $\mathrm{C} 1 / \mathrm{C} 2$ & $\mathrm{C} 1 / \mathrm{C} 2$ & $\mathrm{C} 1 / \mathrm{C} 2$ \\
symmetry & $268 / 536$ & $962 / 1924$ & $972 / 1944$ \\
number of particles & $39 / 35$ & $38 / 33$ & $38 / 22$ \\
estimated resolution $(\AA)$ & & & \\
& & & \\
\hline
\end{tabular}


bioRxiv preprint doi: https://doi.org/10.1101/2021.02.16.431372; this version posted February 17, 2021. The copyright holder for this preprint

(which was not certified by peer review) is the author/funder, who has granted bioRxiv a license to display the preprint in perpetuity. It is made available under aCC-BY 4.0 International license.

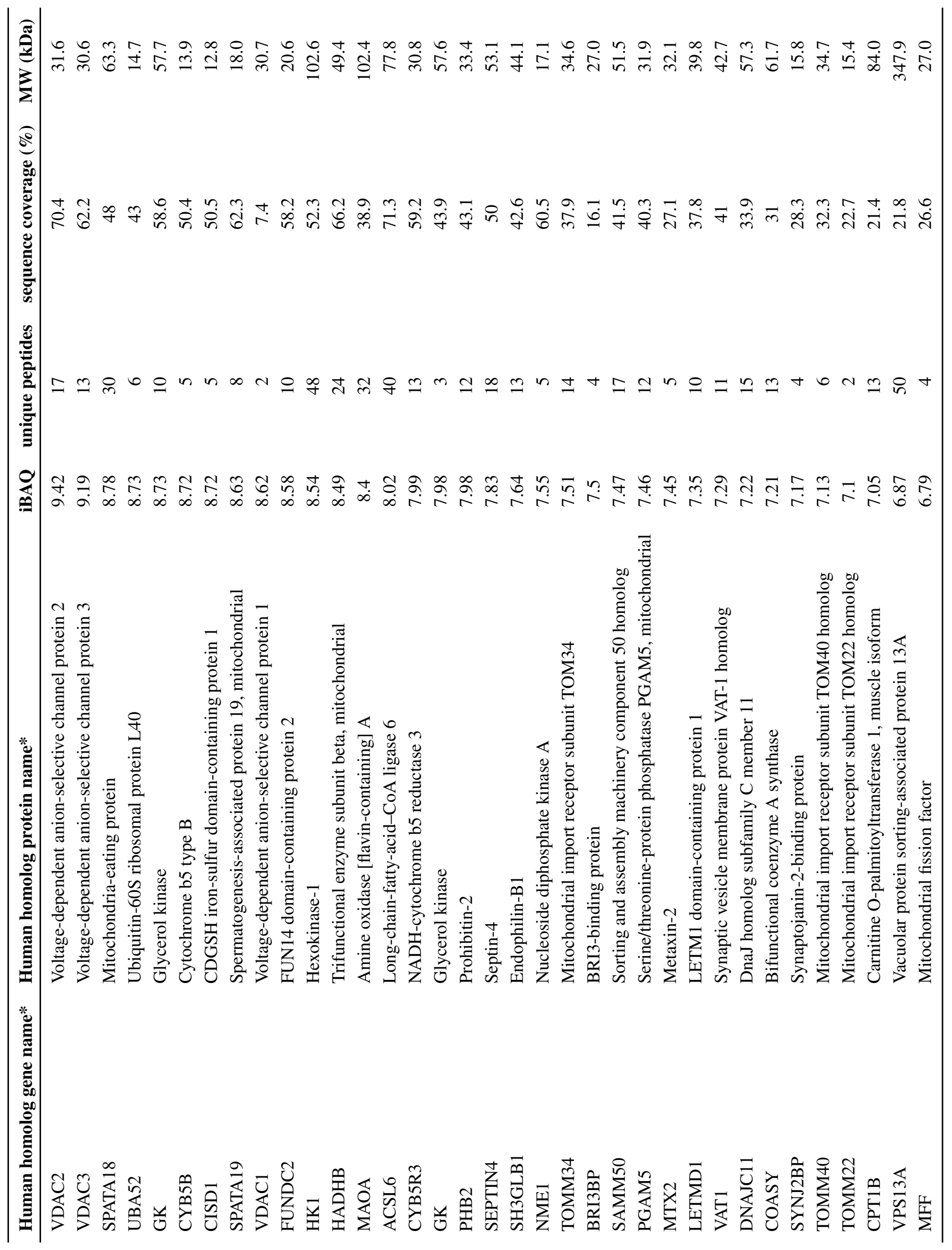


bioRxiv preprint doi: https://doi.org/10.1101/2021.02.16.431372; this version posted February 17, 2021. The copyright holder for this preprint (which was not certified by peer review) is the author/funder, who has granted bioRxiv a license to display the preprint in perpetuity. It is made available under aCC-BY 4.0 International license.

\section{References}

Al-Amoudi, A., Chang, J.-J., Leforestier, A., McDowall, A., Salamin, L.M., Norlén, 802 L.P.O., Richter, K., Blanc, N.S., Studer, D., and Dubochet, J. (2004). Cryo-electron ${ }^{803}$ microscopy of vitreous sections. EMBO J. 23, 3583-3588.

Balogun, E.O., Inaoka, D.K., Shiba, T., Tsuge, C., May, B., Sato, T., Kido, Y., Nara, ${ }^{806}$ T., Aoki, T., Honma, T., et al. (2019). Discovery of trypanocidal coumarins with ${ }^{807}$ dual inhibition of both the glycerol kinase and alternative oxidase of Trypanosoma ${ }^{808}$ brucei brucei. FASEB J. 33, 13002-13013.

Bayrhuber, M., Meins, T., Habeck, M., Becker, S., Giller, K., Villinger, S., Vonrhein, ${ }^{81}$ C., Griesinger, C., Zweckstetter, M., and Zeth, K. (2008). Structure of the human voltage-dependent anion channel. Proc. Natl. Acad. Sci. U. S. A. 105, 15370-15375.

Bystrom, C.E., Pettigrew, D.W., Branchaud, B.P., O'Brien, P., and Remington, S.J. ${ }^{81}$ (1999). Crystal structures of Escherichia coli glycerol kinase variant $S 58 \rightarrow W_{818}^{817}$ in complex with nonhydrolyzable ATP analogues reveal a putative active conformation of the enzyme as a result of domain motion. Biochemistry $38,3508-3518 .{ }^{819}$

Chen, Y., and Sheng, Z.H. (2013). Kinesin-1-syntaphilin coupling mediates ${ }^{821}$ activity-dependent regulation of axonal mitochondrial transport. J. Cell Biol. 202, 351-364.

Chen, M., Dai, W., Sun, S.Y., Jonasch, D., He, C.Y., Schmid, M.F., Chiu, W., and ${ }^{825}$ Ludtke, S.J. (2017a). Convolutional neural networks for automated annotation of ${ }_{827}$ cellular cryo-electron tomograms. Nat. Methods 14, 983-985.

Chen, Y., Liang, P., Huang, Y., Li, M., Zhang, X., Ding, C., Feng, J., Zhang, Z., Zhang, X., Gao, Y., et al. (2017b). Glycerol kinase-like proteins cooperate with Pld6 in regulating sperm mitochondrial sheath formation and male fertility. Cell 832 Discov. 3, 17030 .

Colombini, M. (2012). VDAC structure, selectivity, and dynamics. Biochim. 835 Biophys. Acta - Biomembr. 1818, 1457-1465.

Danev, R., Buijsse, B., Khoshouei, M., Plitzko, J.M., and Baumeister, W. (2014). 838 Volta potential phase plate for in-focus phase contrast transmission electron 839 microscopy. Proc. Natl. Acad. Sci. U. S. A. 111, 15635-15640.

Davila, M.P., Muñoz, P.M., Bolaños, J.M.G., Stout, T.A.E., Gadella, B.M., Tapia, 842 J.A., Balao Da Silva, C., Ortega Ferrusola, C., and Peña, F.J. (2016). Mitochondrial 843 ATP is required for the maintenance of membrane integrity in stallion spermatozoa, 844 whereas motility requires both glycolysis and oxidative phosphorylation. Reproduc- 845 tion 152, 683-694.

Duvert, M., Mazat, J.-P., and Barets, A.-L. (1985). Intermitochondrial junctions in 848 the heart of the frog, Rana esculenta. Cell Tissue Res. 241, 129-137.

Fasci, D., van Ingen, H., Scheltema, R.A., and Heck, A.J.R. (2018). Histone 85 Interaction Landscapes Visualized by Crosslinking Mass Spectrometry in Intact 85 Cell Nuclei. Mol. Cell. Proteomics 17, 2018-2033.

Fawcett, D.W. (1970). A comparative view of sperm ultrastructure. Biol. Reprod. ${ }^{85}$ Suppl. 2, 90-127.

Fawcett, D.W. (1975). The mammalian spermatozoon. Dev. Biol. 44, 394-436.

Fenton, A.R., Jongens, T.A., and Holzbaur, E.L.F. (2021). Mitochondrial dynamics: ${ }^{860}$ Shaping and remodeling an organelle network. Curr. Opin. Cell Biol. 68, 28-36.

Friend, D.S., and Heuser, J.E. (1981). Orderly particle arrays on the mitochondrial ${ }^{863}$ outer membrane in rapidly-frozen sperm. Anat. Rec. 199, 159-175.

Fukuda, Y., Laugks, U., Lučić, V., Baumeister, W., and Danev, R. (2015). Electron ${ }_{867}^{866}$ cryotomography of vitrified cells with a Volta phase plate. J. Struct. Biol. 190, ${ }_{868}^{867}$ 143-154.

Fukuda, Y., Abe, A., Tamura, T., Kishimoto, T., Sogabe, A., Akanuma, S., Yokobori, 87 S.I., Yamagishi, A., Imada, K., and Inagaki, K. (2016). Epistasis effects of 872 multiple ancestral-consensus amino acid substitutions on the thermal stability 873 of glycerol kinase from Cellulomonas sp. NT3060. J. Biosci. Bioeng. 121, 497-502. 874

Glancy, B., Hartnell, L.M., Malide, D., Yu, Z.X., Combs, C.A., Connelly, P.S., ${ }^{875}$ Subramaniam, S., and Balaban, R.S. (2015). Mitochondrial reticulum for cellular 877 energy distribution in muscle. Nature 523, 617-620.

Goddard, T.D., Huang, C.C., Meng, E.C., Pettersen, E.F., Couch, G.S., Morris, J.H., and Ferrin, T.E. (2018). UCSF ChimeraX: Meeting modern challenges in 881 visualization and analysis. Protein Sci. 27, 14-25.

Gonçalves, R.P., Buzhynskyy, N., Prima, V., Sturgis, J.N., and Scheuring, S. (2007). 884 Supramolecular Assembly of VDAC in Native Mitochondrial Outer Membranes. J. 885
Mol. Biol. 369, 413-418.

Grant, B.J., Rodrigues, A.P.C., EISawy, K.M., McCammon, J.A., and Caves, L.S.D. (2006). Bio3d: an R package for the comparative analysis of protein structures. Bioinformatics 22, 2695-2696.

Guo, X.W., and Mannella, C.A. (1993). Conformational change in the mitochondrial channel, VDAC, detected by electron cryo-microscopy. Biophys. J. 64, 545-549.

Gutnick, A., Banghart, M.R., West, E.R., and Schwarz, T.L. (2019). The light-sensitive dimerizer zapalog reveals distinct modes of immobilization for axonal mitochondria. Nat. Cell Biol. 21, 768-777.

Hackenbrock, C.R. (1968). Ultrastructural Bases for Metabolically Linked Mechanical Activity in Mitochondria II. Electron Transport-Linked Ultrastructural Transformations in Mitochondria. J. Cell Biol. 37, 345-369.

Heumann, J.M., Hoenger, A., and Mastronarde, D.N. (2011). Clustering and variance maps for cryo-electron tomography using wedge-masked differences. J. Struct. Biol. 175, 288-299.

Hinsch, K.D., De Pinto, V., Aires, V.A., Schneider, X., Messina, A., and Hinsch, E. (2004). Voltage-dependent Anion-selective Channels VDAC2 and VDAC3 Are Abundant Proteins in Bovine Outer Dense Fibers, a Cytoskeletal Component of the Sperm Flagellum. J. Biol. Chem. 279, 15281-15288.

Ho, H.-C.C., and Wey, S. (2007). Three dimensional rendering of the mitochondrial sheath morphogenesis during mouse spermiogenesis. Microsc. Res. Tech. 70, 719-723.

Hoogenboom, B.W., Suda, K., Engel, A., and Fotiadis, D. (2007). The Supramolecular Assemblies of Voltage-dependent Anion Channels in the Native Membrane. J. Mol. Biol. 370, 246-255.

Huang, X., Sun, L., Ji, S., Zhao, T., Zhang, W., Xu, J., Zhang, J., Wang, Y., Wang, X., Franzini-Armstrong, C., et al. (2013). Kissing and nanotunneling mediate intermitochondrial communication in the heart. Proc. Natl. Acad. Sci. U. S. A. 110, 2846-2851.

Hutcheon, K., McLaughlin, E.A., Stanger, S.J., Bernstein, I.R., Dun, M.D., Eamens, A.L., and Nixon, B. (2017). Analysis of the small non-protein-coding RNA profile of mouse spermatozoa reveals specific enrichment of piRNAs within mature spermatozoa. RNA Biol. 14, 1776-1790.

Imai, H., Hakkaku, N., Iwamoto, R., Suzuki, J., Suzuki, T., Tajima, Y., Konishi, K. Minami, S., Ichinose, S., Ishizaka, K., et al. (2009). Depletion of selenoprotein GPx4 in spermatocytes causes male infertility in mice. J. Biol. Chem. 284, 32522-32532.

Kang, J.S., Tian, J.H., Pan, P.Y., Zald, P., Li, C., Deng, C., and Sheng, Z.H. (2008). Docking of Axonal Mitochondria by Syntaphilin Controls Their Mobility and Affects Short-Term Facilitation. Cell 132, 137-148.

Kim, D.E., Chivian, D., and Baker, D. (2004). Protein structure prediction and analysis using the Robetta server. Nucleic Acids Res. 32, W526-W531.

Klykov, O., Steigenberger, B., Pektaş, S., Fasci, D., Heck, A.J.R., and Scheltema, R.A. (2018). Efficient and robust proteome-wide approaches for cross-linking mass spectrometry. Nat. Protoc. 13, 2964-2990.

Konieczny, P., Fuchs, P., Reipert, S., Kunz, W.S., Zeöld, A., Fischer, I., Paulin, D., Schröder, R., and Wiche, G. (2008). Myofiber integrity depends on desmin network targeting to Z-disks and costameres via distinct plectin isoforms. J. Cell Biol. 181, $667-681$

Kremer, J.R., Mastronarde, D.N., and McIntosh, J.R. (1996). Computer Visualization of Three-Dimensional Image Data Using IMOD. J. Struct. Biol. 116, 71-76.

Kwon, W.S., Park, Y.J., Mohamed, E.S.A., and Pang, M.G. (2013). Voltagedependent anion channels are a key factor of male fertility. Fertil. Steril. 99, 354-361.

Liu, F., Lössl, P., Rabbitts, B.M., Balaban, R.S., and Heck, A.J.R. (2018). The interactome of intact mitochondria by cross-linking mass spectrometry provides evidence for coexisting respiratory supercomplexes. Mol. Cell. Proteomics 17, 216-232.

Mannella, C.A. (1982). Structure of the outer mitochondrial membrane: Ordered arrays of porelike subunits in outer-membrane fractions from neurospora crassa mitochondria. J. Cell Biol. 94, 680-687.

Mannella, C.A. (1998). Conformational changes in the mitochondrial channel protein, VDAC, and their functional implications. J. Struct. Biol. 121, 207-218.

Marin, S., Chiang, K., Bassilian, S., Lee, W.N.P., Boros, L.G., Fernández-Novell, J.M., Centelles, J.J., Medrano, A., Rodriguez-Gil, J.E., and Cascante, M. (2003). 
bioRxiv preprint doi: https://doi.org/10.1101/2021.02.16.431372; this version posted February 17, 2021. The copyright holder for this preprint (which was not certified by peer review) is the author/funder, who has granted bioRxiv a license to display the preprint in perpetuity. It is made available under aCC-BY 4.0 International license.

Metabolic strategy of boar spermatozoa revealed by a metabolomic characteriza- 971 tion. FEBS Lett. 554, 342-346.

De Martino, C., Floridi, A., Marcante, M.L., Malorni, W., Barcellona, P.S., Bellocci, 974 M., and Silvestrini, B. (1979). Morphological, histochemical and biochemical 975 studies on germ cell mitochondria of normal rats. Cell Tissue Res. 196, 1-22. 976

Mastronarde, D.N. (2005). Automated electron microscope tomography using 978 robust prediction of specimen movements. J. Struct. Biol. 152, 36-51. 979

Mederacke, I., Dapito, D.H., Affò, S., Uchinami, H., and Schwabe, R.F. (2015). 981 High-yield and high-purity isolation of hepatic stellate cells from normal and fibrotic 982 mouse livers. Nat. Protoc. 10, 305-315.

Mi, Y., Shi, Z., and Li, J. (2015). Spata19 is critical for sperm mitochondrial function 985 and male fertility. Mol. Reprod. Dev. 82, 907-913.

Milner, D.J., Mavroidis, M., Weisleder, N., and Capetanaki, Y. (2000). Desmin 988 cytoskeleton linked to muscle mitochondrial distribution and respiratory function. J. ${ }^{989}$ Cell Biol. 150, 1283-1297.

Moore, A.S., and Holzbaur, E.L. (2018). Mitochondrial-cytoskeletal interactions: 992 dynamic associations that facilitate network function and remodeling. Curr. Opin. ${ }^{993}$ Physiol. 3, 94-100.

Mukai, C., and Okuno, M. (2004). Glycolysis Plays a Major Role for Adenosine 996 Triphosphate Supplementation in Mouse Sperm Flagellar Movement. Biol. Reprod. ${ }^{997}$ $71,540-547$.

Newport, T.D., Sansom, M.S.P., and Stansfeld, P.J. (2019). The MemProtMD ${ }^{1000}$ database: a resource for membrane-embedded protein structures and their lipid ${ }^{100}$ interactions. Nucleic Acids Res. 47, D390-D397.

Nicastro, D., Schwartz, C., Pierson, J., Gaudette, R., J.R. (2006). The molecular architecture of axonemes revealed by cryoelectron ${ }^{100}$ tomography. Science. 313, 944-948.

Odet, F., Gabel, S., London, R.E., Goldberg, E., and Eddy, E.M. (2013). Glycolysis ${ }^{1008}$ and Mitochondrial Respiration in Mouse LDHC-Null Sperm. Biol. Reprod. 88, 1-7. ${ }_{1010}^{1009}$

Olson, G.E., and Winfrey, V.P. (1986). Identification of a cytoskeletal network ${ }^{1011}$ adherent to the mitochondria of mammalian spermatozoa. J. Ultrastruct. Res. Mol. ${ }_{1013}^{1012}$ Struct. Res. 94, 131-139.

Olson, G.E., and Winfrey, V.P. (1990). Mitochondria-cytoskeleton interactions in the ${ }^{1015}$ sperm midpiece. J. Struct. Biol. 103, 13-22. 1017

Olson, G.E., and Winfrey, V.P. (1992). Structural organization of surface domains ${ }_{1019}^{1018}$ of sperm mitochondria. Mol. Reprod. Dev. 33, 89-98. 1020

Otani, H., Tanaka, O., Kasai, K. -I, and Yoshioka, T. (1988). Development of ${ }_{1022}^{1021}$ mitochondrial helical sheath in the middle piece of the mouse spermatid tail: Regular dispositions and synchronized changes. Anat. Rec. 222, 26-33. 1024

Pan, Y., Decker, W.K., Huq, A.H.H.M., and Craigen, W.J. (1999). Retrotransposition 1025 of Glycerol Kinase-Related Genes from the X Chromosome to Autosomes: 1027 Functional and Evolutionary Aspects. Genomics 59, 282-290.

Perkins, G.A. and Ellisman, M.H. (2011). Mitochondrial configurations in peripheral 1029 nerve suggest differential ATP production. J. Struct. Biol. 173, 117-127. 1031

Pettersen, E.F., Goddard, T.D., Huang, C.C., Couch, G.S., Greenblatt, D.M.,1033 Meng, E.C., and Ferrin, T.E. (2004). UCSF Chimera - A visualization system for ${ }_{1034}$ exploratory research and analysis. J. Comput. Chem. 25, 1605-1612.

Picard, M. McManus, M.J., Csordás, G. Várnai, P. Dorn, G.W. Williams, D. ${ }^{1036}$ Hajnóczky, G., and Wallace, D.C. (2015). Trans-mitochondrial coordination of 1038 cristae at regulated membrane junctions. Nat. Commun. 6, 6259.

Potel, C.M., Lin, M.-H., Heck, A.J.R., and Lemeer, S. (2018). Defeating Major1041 Contaminants in Fe 3+- Immobilized Metal Ion Affinity Chromatography (IMAC) 1042 Phosphopeptide Enrichment. Mol. Cell. Proteomics 17, 1028-1034.

Quillin, M.L., and Matthews, B.W. (2000). Accurate calculation of the density of 1045 proteins. Acta Crystallogr. Sect. D Biol. Crystallogr. 56, 791-794.

Sampson, M.J., Decker, W.K. Beaudet, A. Ruitenbeek, W. Armstrong, D., 1048 Hicks, M.J., and Craigen, W.J. (2001). Immotile Sperm and Infertility in Mice1049 Lacking Mitochondrial Voltage-dependent Anion Channel Type 3. J. Biol. Chem.1050 276, 39206-39212.

Schaffer, M., Mahamid, J., Engel, B.D., Laugks, T., Baumeister, W., and Plitzko, J.M. (2017). Optimized cryo-focused ion beam sample preparation aimed at in situ structural studies of membrane proteins. J. Struct. Biol. 197, 73-82.
Schneider, M., Forster, H., Boersma, A., Seiler, A., Wehnes, H., Sinowatz, F., Neumüller, C., Deutsch, M.J., Walch, A., Angelis, M.H., et al. (2009). Mitochondria glutathione peroxidase 4 disruption causes male infertility. FASEB J. 23, 3233-3242.

Schnick, C., Polley, S.D., Fivelman, Q.L., Ranford-Cartwright, L.C., Wilkinson, S.R., Brannigan, J.A., Wilkinson, A.J., and Baker, D.A. (2009). Structure and non-essential function of glycerol kinase in Plasmodium falciparum blood stages. Mol. Microbiol. 71, 533-545.

Schredelseker, J., Paz, A., López, C.J., Altenbach, C., Leung, C.S., Drexler, M.K., Chen, J.-N., Hubbell, W.L., and Abramson, J. (2014). High Resolution Structure and Double Electron-Electron Resonance of the Zebrafish Voltage-dependent Anion Channel 2 Reveal an Oligomeric Population. J. Biol. Chem. 289, 12566-12577.

Shimada, K., Kato, H., Miyata, H., and Ikawa, M. (2019). Glycerol kinase 2 is essential for proper arrangement of crescent-like mitochondria to form the mitochondrial sheath during mouse spermatogenesis. J. Reprod. Dev. 65, 155-162.

Steen, K., Chen, D., Wang, F., Majumdar, R., Chen, S., Kumar, S., Lombard, D.B., Weigert, R., Zieman, A.G., Parent, C.A., et al. (2020). A role for keratins in supporting mitochondrial organization and function in skin keratinocytes. Mol. Biol. Cell 31, 1103-1111.

Stone, M.R., O'Neill, A., Lovering, R.M., Strong, J., Resneck, W.G., Reed, P.W., Toivola, D.M., Ursitti, J.A., Omary, M.B., and Bloch, R.J. (2007). Absence of keratin 19 in mice causes skeletal myopathy with mitochondrial and sarcolemmal reorganization. J. Cell Sci. 120, 3999-4008.

Tegunov, D., and Cramer, P. (2019). Real-time cryo-electron microscopy data preprocessing with Warp. Nat. Methods 16, 1146-1152.

Tivol, W.F., Briegel, A., and Jensen, G.J. (2008). An Improved Cryogen for Plunge Freezing. Microsc. Microanal. 14, 375-379.

Tomasello, M.F., Guarino, F., Reina, S., Messina, A., and De Pinto, V. (2013). The Voltage-Dependent Anion Selective Channel 1 (VDAC1) Topography in the Mitochondrial Outer Membrane as Detected in Intact Cell. PLoS One 8, e81522.

Tourmente, M., Villar-Moya, P., Rial, E., and Roldan, E.R.S. (2015). Differences in ATP generation via glycolysis and oxidative phosphorylation and relationships with sperm motility in mouse species. J. Biol. Chem. 290, 20613-20626.

Viana, M.P., Brown, A.I., Mueller, I.A., Goul, C., Koslover, E.F., and Rafelski, S.M. (2020). Mitochondrial Fission and Fusion Dynamics Generate Efficient, Robust, and Evenly Distributed Network Topologies in Budding Yeast Cells. Cell Syst. 10 287-297.e5.

Vincent, A.E., Turnbull, D.M., Eisner, V., Hajnóczky, G., and Picard, M. (2017). Mitochondrial Nanotunnels. Trends Cell Biol. 27, 787-799.

Vincent, A.E., White, K., Davey, T., Philips, J., Ogden, R.T., Lawess, C., Warren, C., Hall, M.G., Ng, Y.S., Falkous, G., et al. (2019). Quantitative 3D Mapping of the Human Skeletal Muscle Mitochondrial Network. Cell Rep. 26, 996-1009.e4.

Wessel, D., and Flügge, U.I. (1984). A method for the quantitative recovery of protein in dilute solution in the presence of detergents and lipids. Anal. Biochem. 138, 141-143.

Wolff, G., Limpens, R.W.A.L., Zheng, S., Snijder, E.J., Agard, D.A., Koster, A.J., and Bárcena, M. (2019). Mind the gap: Micro-expansion joints drastically decrease the bending of FIB-milled cryo-lamellae. J. Struct. Biol. 208, 107389.

Woolley, D.M., Neesen, J., and Vernon, G.G. (2005). Further studies on knockout mice lacking a functional dynein heavy chain (MDHC7). 2. A developmental explanation for the asthenozoospermia. Cell Motil. Cytoskeleton 61, 74-82.

Zeng, G.L. (2012). A filtered backprojection algorithm with characteristics of the iterative landweber algorithm. Med. Phys. 39, 603-607.

Zhang, Y., Ou, Y., Cheng, M., Shojaei Saadi, H., Thundathil, J.C., and van der Hoorn, F.A. (2012). KLC3 is involved in sperm tail midpiece formation and sperm function. Dev. Biol. 366, 101-110.

Zheng, S.Q., Palovcak, E., Armache, J.P., Verba, K.A., Cheng, Y., and Agard, D.A. (2017). MotionCor2: Anisotropic correction of beam-induced motion for improved cryo-electron microscopy. Nat. Methods 14, 331-332. 